\title{
Geochemistry of basaltic flows from a basalt ring structure of the Serra Geral formation at Água Vermelha dam, Triângulo Mineiro, Brazil: implications for the magmatic evolution of the Paraná-Etendeka Province
}

\author{
Fernando Estevao Rodrigues Crincoli Pacheco ${ }^{1 *}(\mathbb{D})$, \\ Fabricio de Andrade Caxito ${ }^{1}$ (D), Lucia Castanheira de Moraes $^{2}$ (D), \\ Antonio Carlos Pedrosa-Soares ${ }^{1}$ D , Glaucia Nascimento Queiroga ${ }^{3}$ (D)
}

\begin{abstract}
The Serra Geral Formation belongs to the Parana-Etendeka Magmatic Province (PEMP) and its geochemical and petrographic characteristics are not homogeneous. Many studies segment this group into six basaltic and two rhyolitic magma-types. It is believed that its extrusion occurred through crustal fissures in the Cretaceous, but some authors described the presence of conduits in the shape of basaltic ring structures (BRS) in the Agua Vermelha region in the North of the province. The BRS rocks, based on textures and structures, were divided into four groups-central flow, basal flow, main ring dyke and lava flow - with a very similar petrography, composed of plagioclase (labradorite-bytownite), clinopyroxene (augite) and oxide (titanomagnetite) with intergranular texture. The whole-rock analyses of the basal and lava flows allow classifying them as tholeiitic basalts of the Paranapanema magma-type. Geochemical data interpretation suggests an enriched magma source, with low degree of partial melting, high depth of melt generation and without significant crustal contamination. The BRS experienced fractional crystallization on shallow magma chamber, influenced by successive new injections from different parental magmas which would be responsible for the pulses of effusion and explosion. Thus, the singularities of the BRS of Agua Vermelha are important to comprehend the evolution of the PEMP.
\end{abstract}

KEYWORDS: Paraná-Etendeka Magmatic Province; Serra Geral Formation; lithochemistry; petrography; mineral chemistry.

\section{INTRODUCTION}

The Serra Geral Formation represents thick sequence flows of mainly basaltic rocks (ca. $1,700 \mathrm{~m}$ of maximum thickness), and belongs to the continental-scale Paraná-Etendeka Magmatic Province (PEMP) (Almeida 1986). Due to its extension, its characteristics are not homogeneous. Bimodal magmatism was responsible for predominantly basaltic and subordinate dacitic-rhyolitic rocks found at the province.
It is widely thought that the main extrusion mechanism for this rapid basaltic volcanism of the PEMP was through crustal fissures formed during the Cretaceous, due to the break-up of West Gondwana (Almeida 1986). Dyke swarms are commonly found oriented according to tens of km-long fractures, such as the Ponta Grossa, Serra do Mar and Florianópolis (Marques \& Ernesto 2004). Except the ones of Florianópolis Swarm, whose ages are subject of some debate, the other dykes are slightly younger than the flows (e.g., Deckart et al. 1998, Renne et al.

${ }^{1}$ Programa de Pós-Graduação em Geologia, Universidade Federal de Minas Gerais - Belo Horizonte (MG), Brazil. E-mails: ferodrigues@live.it; caxito@ufmg.br; pedrosa@pq.cnpq.br

${ }^{2}$ Centro Federal de Educação Tecnológica de Minas Gerais - Araxá (MG), Brazil. E-mail: 2013luciam@gmail.com

${ }^{3}$ Departamento de Geologia, Escola de Minas, Universidade Federal de Ouro Preto - Ouro Preto (MG), Brazil. E-mail: glauciaqueiroga@yahoo.com.br

*Corresponding author.

Manuscript ID: 20170084. Received on: 06/30/2017. Approved on: 04/23/2018. 
1996). In other similar provinces, basaltic ring structures (BRS) are eventually found (e.g., Swanson et al. 1975, Jaeger et al. 2005, Webster et al. 2006), and in the Serra Geral Formation, 11 BRS were identified in the northern area of the province and were characterized as possible conduits of lava (Araújo et al. 1977, Araújo 1982, Araújo $\&$ Hasui, 1985). This descriptive term (BRS) refers to rimmed topographic depression within basaltic lava flow which appears in plain view as a circular or an elliptical structure with raised rims (Burr et al. 2009).

In this paper, we present detailed petrographic, lithochemical and mineral chemistry data from the basalts of one of those BRS situated in the Northern portion of the Serra Geral Formation, at the Triângulo Mineiro region, Brazil. This study contributes to a better characterization of the BRS lava flows and to improve the understanding about the geochemical evolution of the Serra Geral Formation, since the BRS might represent a volcanic conduit and its analyses can point the magmatic source characteristics, as well as differentiation and crystallization processes that occurred on PEMP flows.

\section{GEOLOGICAL CONTEXT}

The development of the Paraná basin occurred during the Phanerozoic upon a crystalline and metasedimentary basement in the southeastern region of the South American platform, which was profoundly affected by tectonic, magmatic and metamorphic events during the Neoproterozoic (ca. 900 - $530 \mathrm{Ma}$ ) (Zalán et al. 1991). Deposition of the sedimentary-magmatic sequence that filled the Paraná basin occurred from the Upper Ordovician to the Upper Cretaceous (Milani 2004).

The Serra Geral Formation represents more than $90 \%$ of the preserved part of the Paraná-Etendeka Magmatic Province (PEMP) and its origin is related to the break-up of Gondwana and the opening of the South Atlantic Ocean. The PEMP is composed by a thick volcanic succession which covers a great portion of southern Brazil and parts of Paraguay, Uruguay and Argentina (Marques \& Ernesto 2004) and occupies an area of $9.17 \times 10^{5} \mathrm{~km}^{2}$ with about $1.7 \times 10^{6} \mathrm{~km}^{3}$ of, predominantly, basaltic rocks (Frank et al. 2009), along with mafic sills and dyke swarms that crosscut the sedimentary basin (Milani et al. 2007).

The basic volcanic rocks of the Serra Geral Formation are divided into six magma-types: Urubici, Pitanga and Paranapanema, from the high titanium group $\left(\mathrm{HTi} ; \mathrm{TiO}_{2}\right.$ $>2 \mathrm{wt} \%$ ); and Gramado, Esmeralda and Ribeira, from the low titanium group ( $\mathrm{LTi} ; \mathrm{TiO}_{2}<2 \mathrm{wt} \%$ ). The rhyolitic magma were separated due to the amount of incompatible elements, being the Palmas and Chapecó types depleted and enriched in those elements respectively (e.g., Bellieni et al. 1984, Mantovani et al. 1985, Piccirillo \& Melfi 1988, Peate et al. 1992).

The distribution of those magma-types is not random through the PMPE. Although the Pitanga and Paranapanema types ( $\mathrm{HTi}$ ) occur through the entire province, in volume they are preferentially located at the northern area. The LTi and rhyolitic magmas occur on the south-central part of the province (Janasi et al. 2011) (Fig. 1). The Southern Paraná Magmatic Province hosts the Urubici rocks (HTi) (Piccirillo \& Melfi 1988, Peate 1997), although some scarce flows (Machado et al. 2007) and dykes (Seer et al. 2011, Marques et al. 2016) are found in the northern area.

\section{Água Vermelha Region}

The Água Vermelha region is located between the cities of Iturama (Minas Gerais state) and Ouroeste (São Paulo state), where a hydroelectric dam was constructed over the Grande riverbed. The geological studies in the region date from the time of construction of the dam (e.g., Araújo et al. 1977, Araújo 1982, Araújo \& Hasui 1985). Basaltic rocks of the Serra Geral Formation in the area occur as both dykes and lava flows. The flows are distributed in conspicuous semi-circular structures, while the dykes are disposed in ring structures (Araújo 1982). Also, in the center of one BRS a lava lake structure is

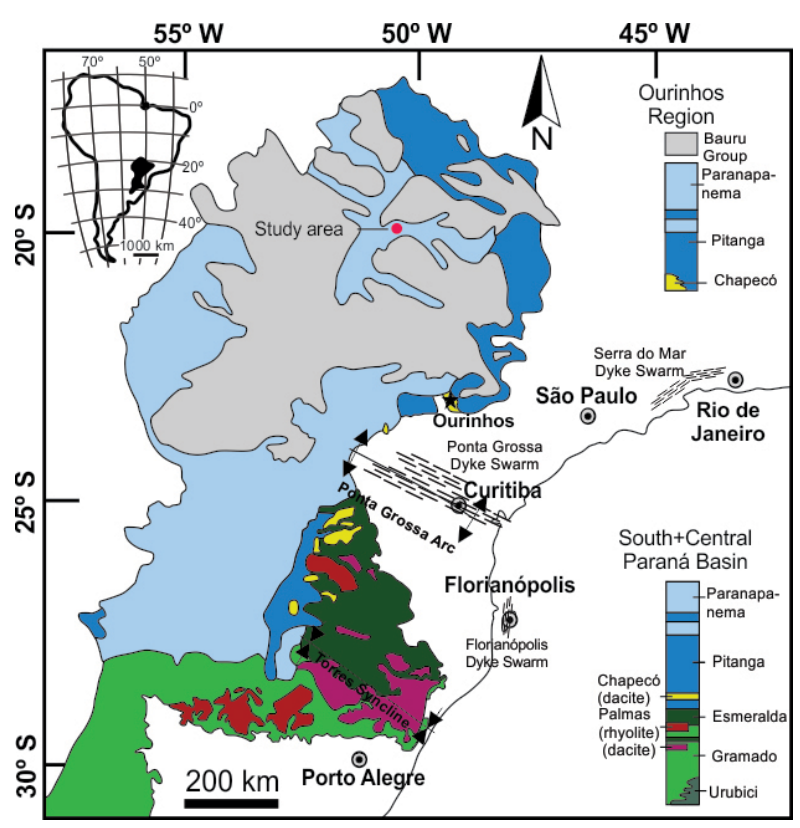

Figure 1. Map of the distribution of magma-types of the Serra Geral Formation throughout the Paraná Basin (adapted from Janasi et al. 2011). The study area is represented by the red dot. 
described, which is surrounded by lava flows and a ring dyke (Pacheco et al. 2018).

Three types of mafic rocks characterize the lava flows described in the region: basaltic breccias, vesicle-amygdaloidal basalts, and massive basalts. The basaltic breccias are restricted and divided into volcanic and pyroclastic types. The semi-circular structures are expressed in the region as depressions and numbered from 1 to 11

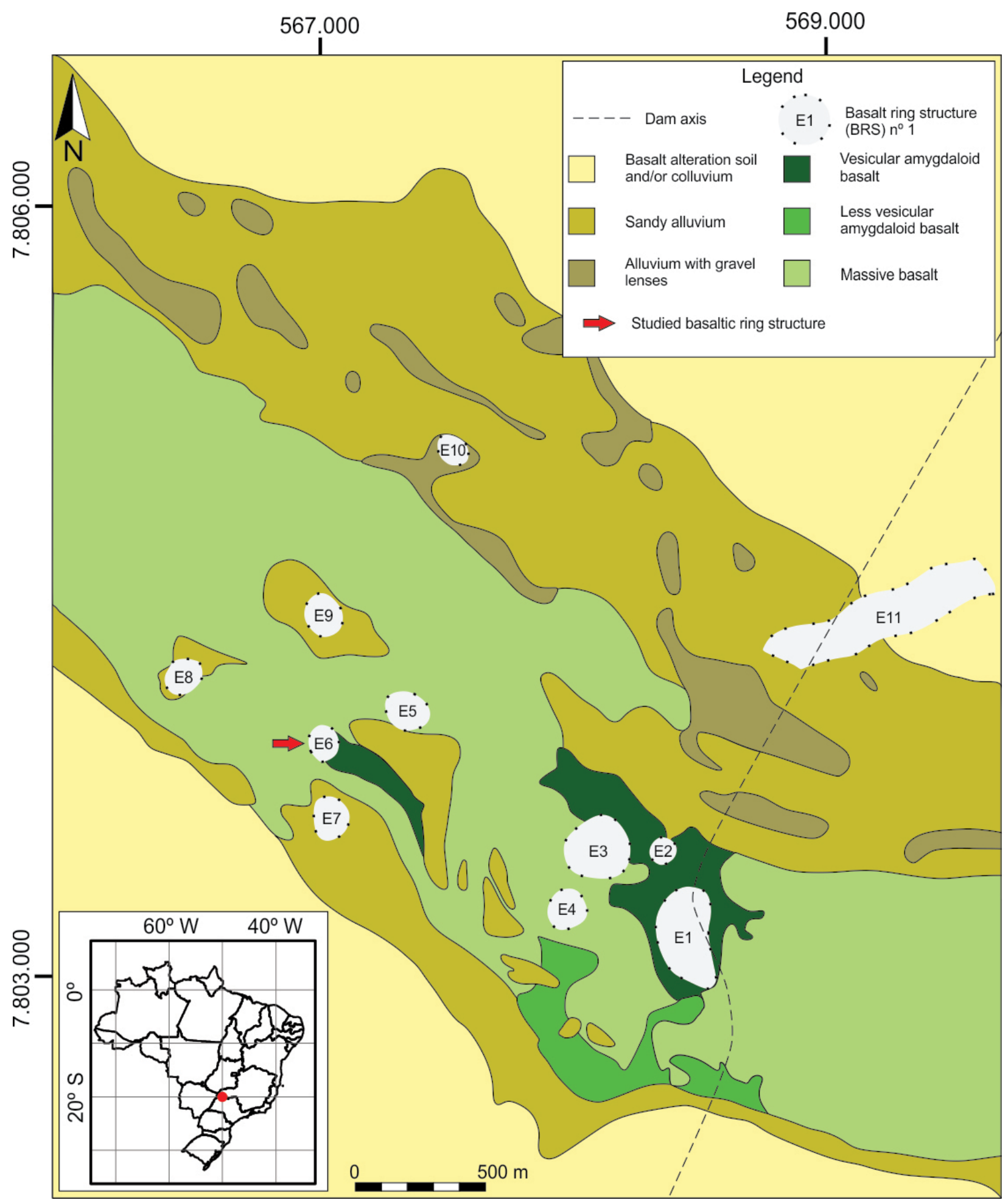

Figure 2. Geological map of the Água Vermelha region (adapted from Araújo 1982). Coordinates are in Universal Transverse Mercator (UTM), World Geodetic System (WGS) 84 Datum. 
in the Figure 2. They are filled by vesicular-amygdaloidal basalts, with pahoehoe structures, and show a sharp contact with neighboring lava flows or ring dyke. Ring fractures are common (Araújo \& Hasui 1985).

\section{MATERIALS AND METHODS}

To characterize each flow of the BRS (Fig. 3), a petrographic study was made with samples of each level. Thirty thin sections to detail the texture and mineralogical aspects and five polished sections were made for mineral chemistry analyses. The samples used for chemical analyses (both mineral chemistry and lithochemistry) are represented on the stratigraphic sections (Fig. 4).

The microanalysis of plagioclase, pyroxene and titanomagnetite were performed with an electron microprobe JEOL JXA-8900 RL at the Microscopy and Microanalysis Laboratory of the Centro de Desenvolvimento da Tecnologia Nuclear (CDTN), at the Federal University of Minas Gerais (Universidade Federal de Minas Gerais - UFMG). The electron beam was set at $15 \mathrm{kV}, 20 \mathrm{nA}$, 2-5 $\mu \mathrm{m}$, and the common matrix $\mathrm{ZAF}-\mathrm{Z}$ : atomic number, A: absorption; F: fluorescence - corrections were applied. Counting times on the peaks/background were $10 / 5 \mathrm{~s}$ for all elements ( $\mathrm{Si}, \mathrm{Na}, \mathrm{Mg}, \mathrm{Mn}, \mathrm{K}, \mathrm{Al}, \mathrm{Fe}$, $\mathrm{Ca}, \mathrm{Ti})$, except for $\mathrm{Cr}$ and $\mathrm{P}(20 / 10 \mathrm{~s})$. Analytical errors are within 0.12 and $1.23 \%$. Plagioclase and clinopyroxene were analyzed along granular spots and from core and rims. Table 1 summarizes the main features of the analysis, as the examined elements and standards. The mineral formulas were calculated based on 6 oxygens for pyroxene and 8 for plagioclase crystals. The total iron content obtained by the microprobe was considered as $\mathrm{FeO}$. The binary and ternary diagrams used to characterize the main minerals were obtained by Microsoft Excel and GCDKit version 2.3.

The whole rock chemical analyses preparation consisted of the crushing and pulverization of ca. $300 \mathrm{~g}$ of homogeneous and unweathered sample on a tungsten carbide shatterbox at the Sample Preparation Laboratory of the Centro de Pesquisas Professor Manoel Teixeira da Costa at Instituto de Ciências (CPMTC-IGC) of UFMG. The sample analyses followed the induced coupled plasma (ICP) routine at SGS Geosol Laboratories. The major elements were analyzed by induced coupled plasma with optical emission spectroscopy (ICP-OES), and the minor and trace elements by induced coupled plasma with mass spectrometry (ICP-MS). The accuracy and precision were better than $10 \%$ and the confidence level was $95 \%$.
The major element variation diagrams and the CIPW norm (Cross et al. 1903) were made after normalization on water-free basis (Gill 2010). The CIPW norm of the standard mineral components, from the whole-rock analyses, was based on Johannsen (1931). Since the whole-rock chemical analyses considered only $\mathrm{Fe}_{2} \mathrm{O}_{3}$, the estimation of $\mathrm{FeO}$ and $\mathrm{Fe}_{2} \mathrm{O}_{3}$ was based on Gill (2010), who used the ratio 0.9, that is, $\mathrm{FeO}$ estimated $=0.9 \times \mathrm{Fe}_{2} \mathrm{O}_{3 \text { Total }}$.

\section{PETROGRAPHY}

The rocks of the BRS were divided in flows due to the easily identifiable top and basal sharp contact of each flow and are represented in the geological map (Fig. 3, Pacheco et al. 2018) and stratigraphic columns (Fig. 4, Pacheco et al. 2018). The central flow occurs in the center of the BRS, with vesicle-amygdaloidal basalts, with spatter and degassing structures. The basal flow represents massive basalt, which shows no structure as columns and has quartz geodes. Seated on it massive lava flows occur, with vertical columnar disjunction structures. The nomenclature of each lava flow follows the numeric order of superposition and lateral continuity. In case in which it was not possible to determine the lateral correlation of each flow, a new sequence was adopted, resulting in two different numberings: 0 to 8 and $1 \mathrm{~A}$ to $3 \mathrm{~A}$; both occurring above the basal flow. The main ring dykes show massive basalt with horizontal to sub-horizontal columnar disjunction structures and crosscut the basal and lava flows.

\section{Central flow}

The central flow is composed of grayish vesicle-amygdaloidal basalt, which is orange when weathered (Fig. 5A). In thin sections, the sample has a predominant intergranular texture with a smaller amount of glass between the crystals. The plagioclase laths are euhedral to subhedral with size of $0.2-0.8 \mathrm{~mm}$ showing Carlsbad twinning, and the clinopyroxene crystals are granular and smaller than $0.5 \mathrm{~mm}$. The amygdala is filled with tabular zeolite crystals $(0.1-0.5 \mathrm{~mm})$ and calcite matrix (Fig. 5B).

It is possible to identify spatter structures of variable size, milimetric to centimetric, reaching up to $15 \mathrm{~cm}$ long (Fig. 5C). The spatter structure has a vitreous matrix and shows larger plagioclase laths $(0.5-1.5 \mathrm{~mm})$ than the intergranular vesicle-amygdaloidal matrix $(0.2-0.8 \mathrm{~mm})$. The plagioclase shows "swallow-tail" endings (Fig. 5D).

The degassing pipes structures reach $15 \mathrm{~cm}$ of diameter (Fig. 5E). In thin sections, they show a vitreous matrix with plagioclase laths smaller than $1 \mathrm{~mm}$, granular 

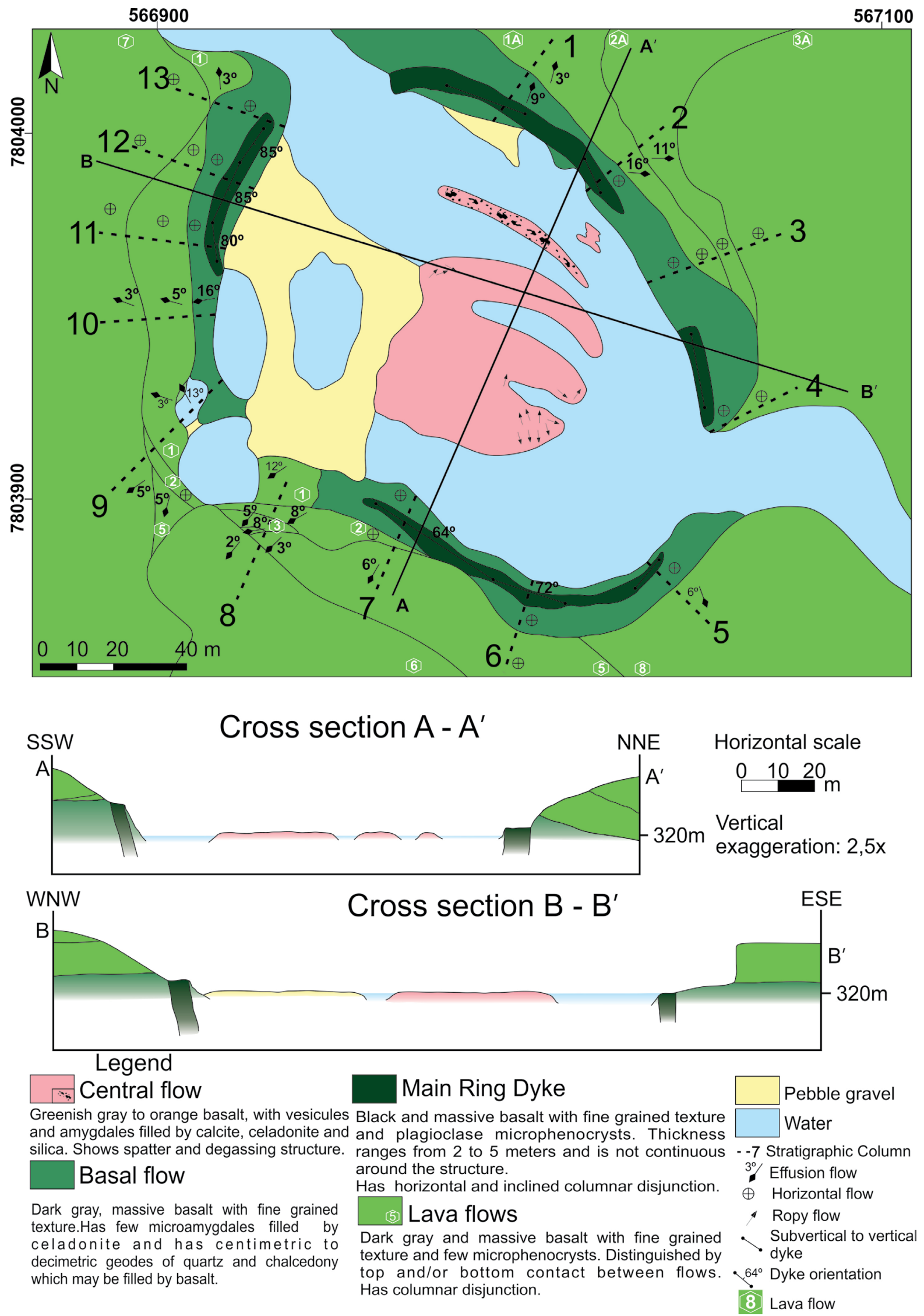

Figure 3. Geological map of basalt ring structure E6 in Água Vermelha region, Minas Gerais / São Paulo, Brazil, showing the different basalt flows, structures and location of the studied stratigraphic sections (Pacheco et al. 2018). 
clinopyroxene crystals smaller than $0.4 \mathrm{~mm}$ and amygdala filled with zeolite (Fig. 5F).

\section{Basal flow}

The basal flow is composed of homogeneous and massive dark grey basalt, with fine-grained plagioclase and pyroxene. It may rarely show some microamygdales $(1-2 \mathrm{~mm})$ filled by celadonite, and towards the top of the flow centimetric to decametric quartz geodes occur, reaching up to $60 \mathrm{~cm}$ in diameter (Fig. $6 \mathrm{~A}$ ). In thin section, it has a predominantly intergranular texture with a smaller amount of glass between the crystals. The plagioclase laths are euhedral to subhedral, smaller than $0.3 \mathrm{~mm}$, showing Carlsbad twinning, and the clinopyroxene crystals are granular and smaller than $0.1 \mathrm{~mm}$. Microphenocrysts of plagioclase forming glomeroporphyritic aggregates are occasionally observed, with $0.5-1.0$ $\mathrm{mm}$ in size, showing Carlsbad twinning and concentric zoning (Fig. 6B). Iddingsite can be found as an olivine pseudomorph and opaque minerals (oxides) occur, with cubic, prismatic and skeletal habit and smaller than 0.1 mm (Figs. 6C and 6D).

\section{Main ring dyke}

It is composed of black basalt with thickness from 2 to $5 \mathrm{~m}$, showing inclined to horizontal columnar disjunctions and it is discontinuous throughout the structure (Fig $6 \mathrm{E})$. In thin section, it has a predominantly intergranular texture with a smaller amount of glass between the crystals. The plagioclase laths are euhedral to subhedral, with size of 0.1 to $0.8 \mathrm{~mm}$, showing Carlsbad twinning and "swallow-tail" endings. The pyroxene crystals are granular and smaller than $0.1 \mathrm{~mm}$. Microamygdales (smaller than $1 \mathrm{~mm}$ ) are filled with clay mineral (Fig. 6F).

Table 1. Overview of the major element set-up for clinopyroxene, plagioclase and titanomagnetite analysis.

\begin{tabular}{l|c|c|c}
\hline Elements & Energetic line & Crystal & Standard \\
\hline $\mathrm{Si}$ & $\mathrm{K} \alpha$ & $\mathrm{TAP}$ & Quartz \\
\hline $\mathrm{Na}$ & $\mathrm{K} \alpha$ & $\mathrm{TAP}$ & Anortoclase \\
\hline $\mathrm{Cr}$ & $\mathrm{K} \alpha$ & $\mathrm{LIF}$ & $\mathrm{Cr}_{2} \mathrm{O}_{3}$ \\
\hline $\mathrm{P}$ & $\mathrm{K} \alpha$ & $\mathrm{PET}$ & Apatite \\
\hline $\mathrm{Mg}$ & $\mathrm{K} \alpha$ & $\mathrm{TAP}$ & MgO \\
\hline $\mathrm{Mn}$ & $\mathrm{K} \alpha$ & $\mathrm{LIF}$ & $\mathrm{Mn}$-Hortonolite \\
\hline $\mathrm{K}$ & $\mathrm{K} \alpha$ & $\mathrm{PET}$ & Anortoclase \\
\hline $\mathrm{Al}$ & $\mathrm{K} \alpha$ & $\mathrm{TAP}$ & Corindon \\
\hline $\mathrm{Fe}$ & $\mathrm{K} \alpha$ & $\mathrm{LIF}$ & Magnetite \\
\hline $\mathrm{Ca}$ & $\mathrm{K} \alpha$ & $\mathrm{PET}$ & Apatite \\
\hline $\mathrm{Ti}$ & $\mathrm{K} \alpha$ & PET & Rutile \\
\hline
\end{tabular}

TAP: thallium acid phthalate crystal; LIF: lithium fluoride crystal; PET: pentaerythritol crystal.

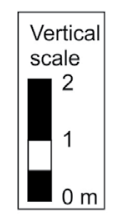

$\hat{\imath}$ Geochemistry

W sample

Microprobe

sample

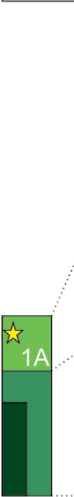

1

the lithochemical and microprobe samples analyzed. The location of each column is represented in the geological map of Fig. 3 by a dashed line. Column number 1 is in the Northern part of the area, and the following columns were made clockwise direction until number 13 (adapted from Pacheco et al. 2018). 


\section{Lava flows}

The lava flows are composed of massive dark grey basalts, with fine phaneritic to aphanitic texture, with rare microphenocrysts. They are separated by sharp top and base contacts and show columnar disjunctions (Fig. 7A).

In thin section, the rock has a subophitic texture with a small amount of glass between the crystals. The matrix has plagioclase laths smaller than $0.5 \mathrm{~mm}$, with Carlsbad twinning and "swallow-tail" endings and granular pyroxene smaller than $0.2 \mathrm{~mm}$. The rock presents microphenocrysts of plagioclase (1-2 $\mathrm{mm})$, which can show Carlsbad twinning, concentric and hourglass zoning (Figs. 7B, 7C and 7D). Microphenocrysts of plagioclase and pyroxene forming glomeroporphyritic aggregates are occasionally observed, with $0.5-1.2 \mathrm{~mm}$ in size (Fig. 7E). Iddingsite can be found as an olivine pseudomorph and opaque minerals (oxide) occur, with cubic and prismatic habits and smaller than $0.1 \mathrm{~mm}$.

\section{LITHOCHEMISTRY}

The major, minor and trace element analysis and the CIPW norm of 14 samples are presented in Table 2. Those data were used to elaborate diagrams which assisted the lithochemistry interpretation of the studied rocks, being 11 samples from the lava flow (LF) and three samples from the basal flow (BF).

The samples did not suffer any significant post-magmatic alteration as indicated by their loss on ignition (LOI) contents $(<1 \mathrm{wt} \%)$ and are classified as basic rocks $\left(\mathrm{SiO}_{2}=48.02-50.65 \mathrm{wt} \%\right)$. The content of alkali elements
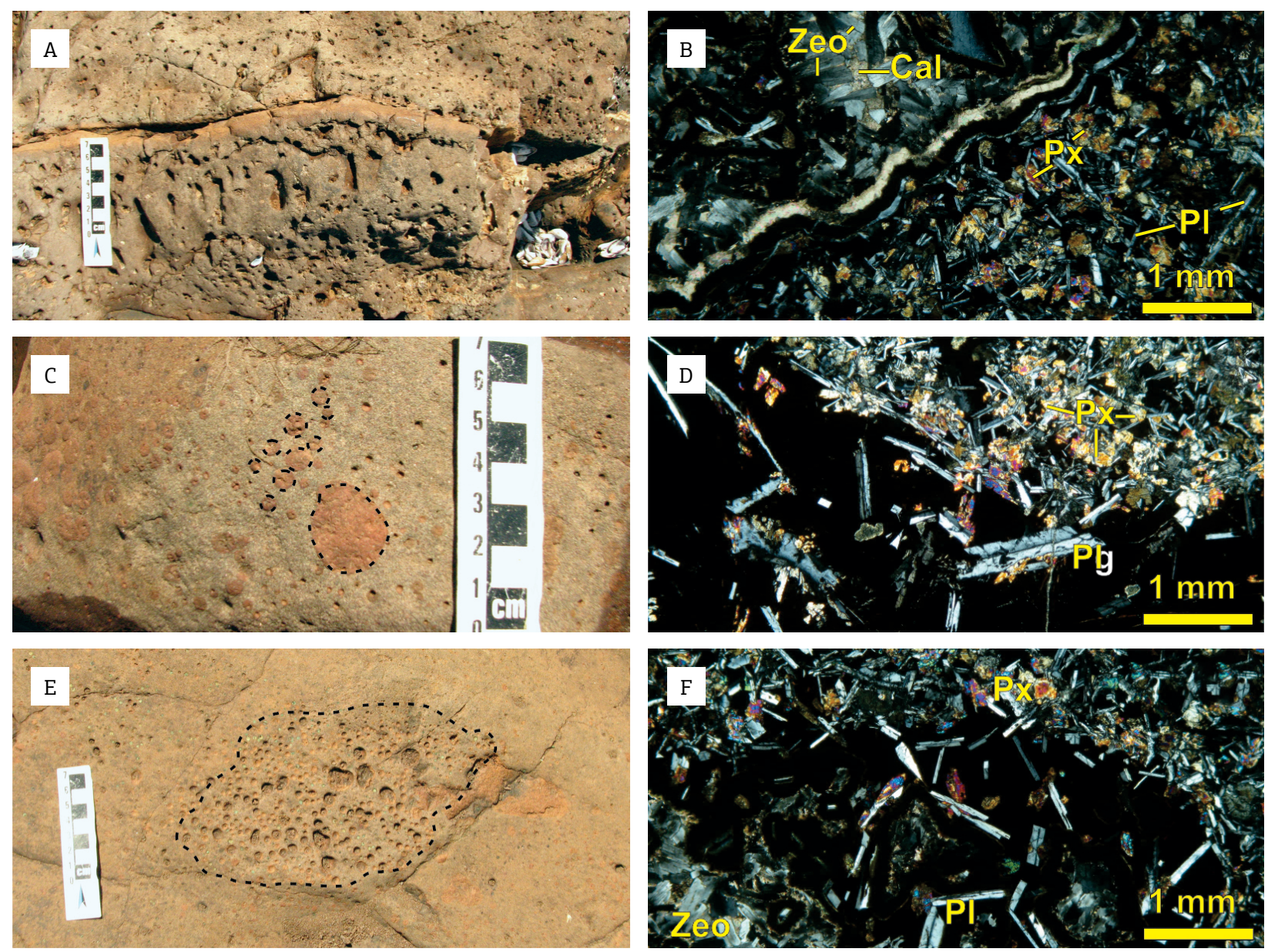

Pl: plagioclase; Px: pyroxene; Zeo: zeolite; Cal: calcite.

Figure 5. (A) Central flow vesicle-amygdaloidal basalt; (B) photomicrography of the central lava flow, with intergranular texture and amygdala filled by zeolite and calcite; (C) spatter structure; (D) photomicrography of spatter structure well-marked by glass and laths; $(E)$ degassing pipe structure; $(F)$ photomicrography of the degassing pipe structure with plagioclase and clinopyroxene laths wrapped by glass and amygdales filled by zeolites. All photomicrographs under crossed polarizers. 
$\left(\mathrm{Na}_{2} \mathrm{O}+\mathrm{K}_{2} \mathrm{O}=3.06-3.28 w t \%\right), \mathrm{Al}_{2} \mathrm{O}_{3}(12.62-13.62$ wt $\%), \mathrm{Fe}_{2} \mathrm{O}_{3} \mathrm{~T}$ (13.83-15.04 wt\%), $\mathrm{MgO}$ (5.56-6.28 wt $\%)$ and $\mathrm{CaO}(9.76-10.58 \mathrm{wt} \%)$ are within the range for basaltic rocks. Those values were calculated on anhydrous basis.

All samples plot on the subalkaline basalt field in the total alkalis/silica (TAS) diagram (Le Maitre 2002), within the field of tholeiitic basalts (MacDonald \& Katsura 1964) (Fig. 8A). The basalts of Água Vermelha belong to the high titanium group $\left(1.96 \mathrm{wt} \%<\mathrm{TiO}_{2}<\right.$ $2.14 \mathrm{wt} \%$ ) according to the magma-type classification of Peate et al. (1992). The $\mathrm{Sr}$ vs. $\mathrm{TiO}_{2}$ and $\mathrm{Ti} / \mathrm{Y}$ vs. Sr diagrams (Peate et al. 1992, Machado et al. 2007) show that all of the samples plot within the Paranapanema field (Figs. $8 \mathrm{~B}$ and $8 \mathrm{C}$ ). It is possible to identify a cluster of samples between the primordial mantle following the path to garnet in residue based on the $(\mathrm{Th} / \mathrm{Nb})_{\mathrm{PM}}$ vs. $(\mathrm{Sm} / \mathrm{Yb})_{\mathrm{PM}}$ ratios (Wang et al. 2007). Although two samples follow the path of crustal contamination, the behavior of other incompatible trace elements, such as $\mathrm{Rb}, \mathrm{Ba}$ and $\mathrm{K}$, does not confirm this petrogenetic process (Fig. 8D).

The covariation of major and trace incompatible elements can be seen through bivariate diagrams using $\mathrm{MgO}$ as an index for differentiation. The determination coefficients of major elements ( $\mathrm{R}^{2}$, adjustment measure of a
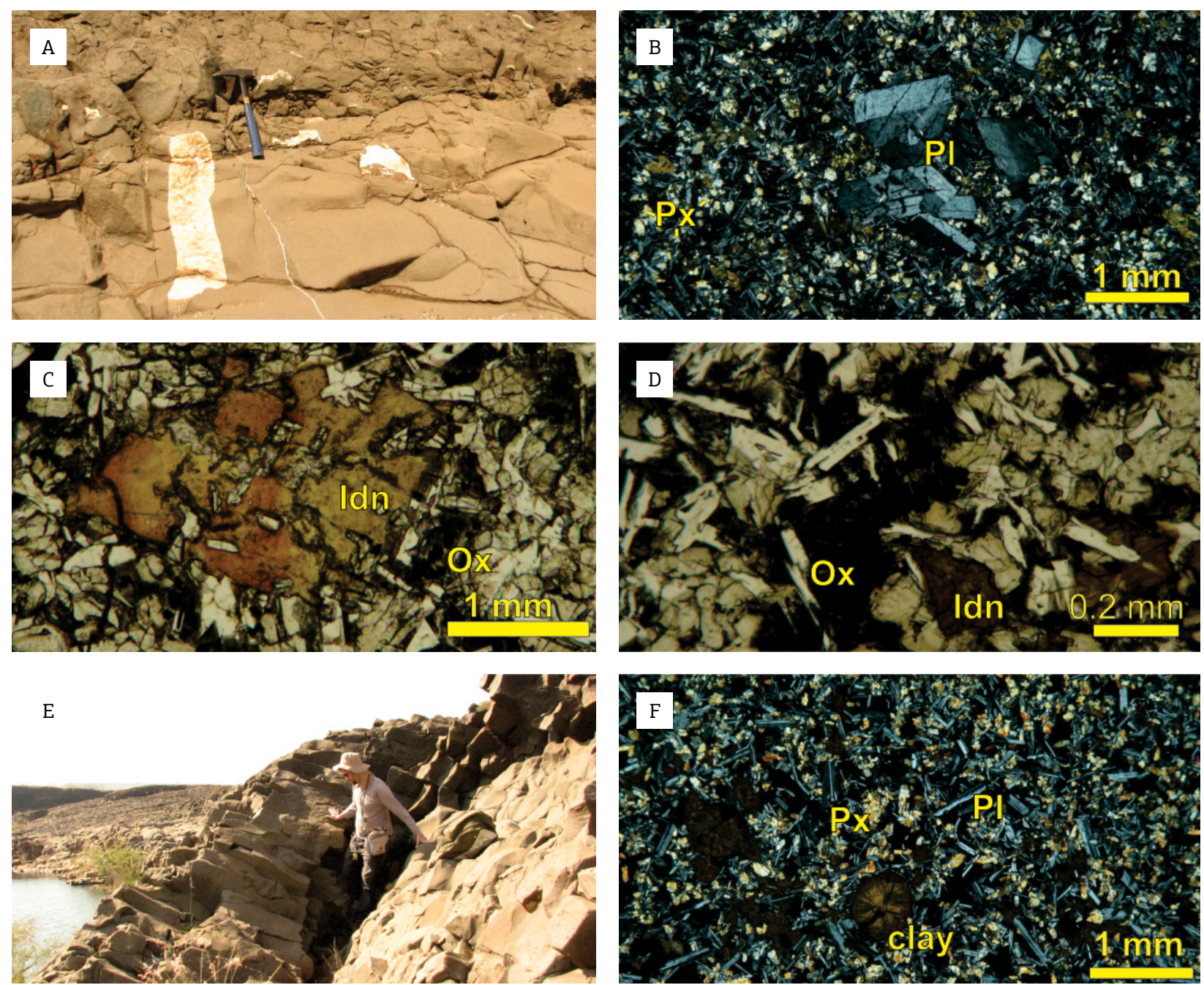

Pl: plagioclase; Px: pyroxene; Idn: iddingsita; Ox: oxide.

Figure 6. (A) Basal flow general aspect, with quartz geode. Photomicrographs of the basal flow basalt, showing glomeroporphyritic aggregate of plagioclase (B), iddingsite and oxides (C and D). (E) Main ring dyke basalt with inclined columnar disjunction; $(\mathrm{F})$ main ring dyke photomicrography with plagioclase and pyroxene laths, glass and microamygdales filled with clay. Photomicrographs B and $F$ under crossed polarizers, and $C$ and $D$ under parallel polarizers. A and E from Pacheco et al. (2018). 
general statistical model) show moderate $\left(31 \%, \mathrm{FeO}_{\mathrm{T}}\right.$; $\left.49 \%, \mathrm{TiO}_{2} ; 51 \%, \mathrm{SiO}_{2}\right)$ and high $(72 \%$ to $\mathrm{Al})$ values, and, when analyzing trace incompatible elements, they show low (<20\%, Rb, Ba, La, U, Th) and moderate (54\%, Sr) values (Fig. 9). It suggests the occurrence of complex differentiation processes during magma evolution.

Both large-ion lithophile elements (LILE) and high field strength elements (HFSE) are enriched when normalized to the primitive mantle (Fig. 10A). Among the LILE, there is a negative $\mathrm{Sr}$ anomaly common to all samples, and among the HFSE there is a negative $\mathrm{Nb}$ anomaly. Other elements show very similar pattern. The $(\mathrm{Rb} /$ $\mathrm{Ba})_{\mathrm{PM}}$ ratio have a strong negative anomaly $(0.54-0.8)$, pointing that the crustal contamination did not take place on the lava ascension (Marques et al. 2017).

The rare earth elements (REE), when normalized to the chondrite (Sun \& McDonough 1989), show enrichment on the total elemental concentration, higher on the light rare earth elements (LREE) and lower on the heavy rare earth elements $(\mathrm{HREE})-(\mathrm{La} / \mathrm{Yb})_{\mathrm{N}}=4.34-6.14$ and $(\mathrm{La} / \mathrm{Sm})_{\mathrm{N}}=2.31-3.02-($ Fig. $10 \mathrm{~B})$, and a negative Eu anomaly $\left(\mathrm{Eu} / \mathrm{Eu}^{*}=0.85-0.94\right)$. When those analyses are compared to Pinto \& Hartmann (2011) data for the Paranapanema type (gray field on Fig. 10B), the patterns are very similar. This enrichment could be enabled by the fractional crystallization of the magma.
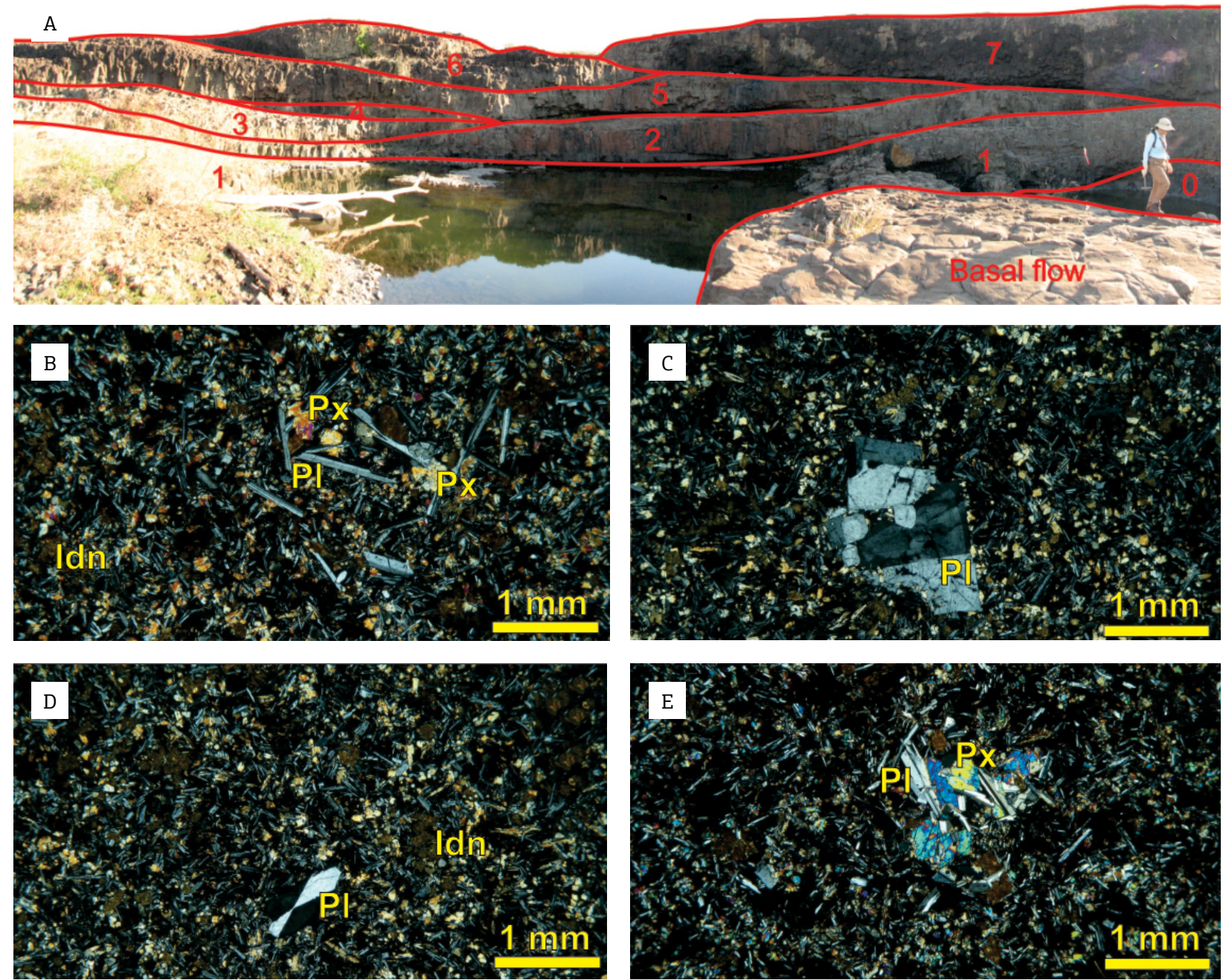

Pl: plagioclase; Px: pyroxene; Idn: iddingsita; Ox: oxide.

Figure 7. (A) Lava flows of the southwestern edge of the mapped ring structure. Universal Transverse Mercator (UTM) coordinates: 7803986 N/567020 E/Zone 22K/ facing southwest (Pacheco et al. 2018). (B-E) Photomicrographs of the lava flow, showing phenocrysts of plagioclase and pyroxene (B), plagioclase with concentric (C) and hourglass zoning (D), and glomeroporphyritic aggregates of plagioclase and pyroxene. All photomicrographs under crossed polarizers. 
Geochemistry of a basaltic ring structure, Serra Geral Fm.

Table 2. Whole-rock analyses of basalts from the ring structure E6 and CIPW norm data.

\begin{tabular}{|c|c|c|c|c|c|c|c|}
\hline Sample & 001 & 002 & 003b & 004 & 005 & 006 & 007 \\
\hline Level & LF 1 & LF 3 & LF 4 & LF 5 & LF 6 & BF & LF 2 \\
\hline North & $7,803,907$ & $7,803,933$ & $7,803,933$ & $7,803,884$ & $7,803,881$ & $7,803,986$ & $7,803,986$ \\
\hline East & 566,934 & 566,906 & 566,906 & 566,898 & 566,933 & 567,020 & 567,020 \\
\hline \multicolumn{8}{|c|}{ Major elements (wt \%) } \\
\hline $\mathrm{SiO}_{2}$ & 50.35 & 48.49 & 49.25 & 48.79 & 49.53 & 48.58 & 49.87 \\
\hline $\mathrm{TiO}_{2}$ & 2.01 & 1.99 & 1.97 & 2.01 & 2.13 & 2.02 & 2.02 \\
\hline $\mathrm{Al}_{2} \mathrm{O}_{3}$ & 13.46 & 13.08 & 13.12 & 13.41 & 13.37 & 13.22 & 13.46 \\
\hline $\mathrm{Fe}_{2} \mathrm{O}_{3}(\mathrm{t})$ & 14.23 & 14.13 & 13.99 & 14.08 & 14.96 & 14.55 & 14.28 \\
\hline $\mathrm{MnO}$ & 0.21 & 0.20 & 0.20 & 0.20 & 0.20 & 0.21 & 0.22 \\
\hline $\mathrm{MgO}$ & 6.03 & 5.86 & 5.87 & 5.96 & 6.01 & 6.22 & 6.00 \\
\hline $\mathrm{CaO}$ & 10.49 & 10.13 & 10.29 & 10.2 & 10.27 & 10.33 & 10.51 \\
\hline $\mathrm{Na}_{2} \mathrm{O}$ & 2.67 & 2.68 & 2.66 & 2.67 & 2.78 & 2.48 & 2.68 \\
\hline $\mathrm{K}_{2} \mathrm{O}$ & 0.57 & 0.48 & 0.52 & 0.49 & 0.48 & 0.55 & 0.54 \\
\hline $\mathrm{P}_{2} \mathrm{O}_{5}$ & 0.21 & 0.21 & 0.22 & 0.22 & 0.22 & 0.21 & 0.22 \\
\hline $\mathrm{Cr}_{2} \mathrm{O}_{3}$ & 0.01 & 0.01 & 0.01 & 0.01 & 0.01 & 0.01 & 0.01 \\
\hline LOI & 0.60 & 0.55 & 0.68 & 0.74 & 0.53 & 0.94 & 0.64 \\
\hline Total & 100.86 & 97.81 & 98.79 & 98.79 & 100.5 & 99.34 & 100.46 \\
\hline
\end{tabular}

Minor and trace elements (ppm)

\begin{tabular}{c|c|c|c|c|c|c|c}
\hline $\mathrm{Zn}$ & 90 & 97 & 90 & 87 & 93 & 90 & 90 \\
\hline $\mathrm{Cu}$ & 234 & 237 & 233 & 232 & 238 & 226 & 229 \\
\hline $\mathrm{Ni}$ & 69 & 72 & 68 & 64 & 64 & 66 & 67 \\
\hline $\mathrm{Ba}$ & 286 & 263 & 265 & 265 & 266 & 283 & 258 \\
\hline $\mathrm{Cs}$ & 0.36 & 0.35 & 0.24 & 0.17 & 0.3 & 0.45 & 0.22 \\
\hline $\mathrm{Ga}$ & 20.5 & 20.7 & 20.5 & 20.7 & 20.9 & 20.1 & 20.4 \\
\hline $\mathrm{Hf}$ & 4.73 & 9.13 & 12.5 & 3.94 & 4.04 & 3.74 & 3.9 \\
\hline $\mathrm{Nb}$ & 20.34 & 17.25 & 21.18 & 12.1 & 12.91 & 16.31 & 11.93 \\
\hline $\mathrm{Rb}$ & 20.3 & 16.3 & 14.8 & 14.7 & 15.5 & 15.3 & 17.1 \\
\hline $\mathrm{Sn}$ & 3.1 & 5.1 & 8.2 & $<0.3$ & 2.7 & 0.5 & 0.5 \\
\hline $\mathrm{Sr}$ & 312 & 299 & 306 & 303 & 300 & 309 & 308 \\
\hline $\mathrm{Th}$ & 4.5 & 9.7 & 14.7 & 2.7 & 2.9 & 3.4 & 2.6 \\
\hline $\mathrm{U}$ & 0.61 & 1.03 & 2 & 0.56 & 0.57 & 1.05 & 0.53 \\
\hline $\mathrm{V}$ & 426 & 415 & 427 & 413 & 412 & 400 & 412 \\
\hline $\mathrm{Zr}$ & 128 & 194 & 230 & 114 & 128 & 114 & 126 \\
\hline $\mathrm{Y}$ & 29.74 & 30.02 & 31.45 & 29.16 & 29.93 & 28.17 & 28.82 \\
\hline $\mathrm{La}$ & 23.6 & 21.7 & 22.2 & 25.7 & 20.9 & 20.1 & 22 \\
\hline $\mathrm{Ce}$ & 43.9 & 43.5 & 43.5 & 43.8 & 43.1 & 41.3 & 42.4 \\
\hline
\end{tabular}


Table 2. Continuation.

\begin{tabular}{c|c|c|c|c|c|c|c}
\hline Sample & $\mathbf{0 0 1}$ & $\mathbf{0 0 2}$ & $\mathbf{0 0 3 b}$ & $\mathbf{0 0 4}$ & $\mathbf{0 0 5}$ & $\mathbf{0 0 6}$ & $\mathbf{0 0 7}$ \\
\hline Level & LF 1 & LF 3 & LF 4 & LF 5 & LF 6 & BF & LF 2 \\
\hline $\mathrm{Pr}$ & 5.68 & 5.57 & 5.62 & 5.52 & 5.59 & 5.35 & 5.48 \\
\hline $\mathrm{Nd}$ & 22.5 & 22.7 & 23 & 22.7 & 22.8 & 21.9 & 22.2 \\
\hline $\mathrm{Sm}$ & 5.5 & 5.6 & 5.6 & 5.5 & 5.6 & 5.4 & 5.3 \\
\hline $\mathrm{Eu}$ & 1.71 & 1.71 & 1.67 & 1.69 & 1.72 & 1.62 & 1.7 \\
\hline $\mathrm{Gd}$ & 5.73 & 6.06 & 5.91 & 5.82 & 5.81 & 5.62 & 5.63 \\
\hline $\mathrm{Tb}$ & 0.94 & 0.96 & 1 & 0.9 & 0.92 & 0.88 & 0.89 \\
\hline $\mathrm{Dy}$ & 5.88 & 5.96 & 6.4 & 5.73 & 5.77 & 5.59 & 5.69 \\
\hline $\mathrm{Ho}$ & 1.16 & 1.21 & 1.35 & 1.15 & 1.15 & 1.12 & 1.12 \\
\hline $\mathrm{Er}$ & 3.28 & 3.47 & 4.06 & 3.12 & 3.26 & 3.05 & 3.12 \\
\hline $\mathrm{Tm}$ & 0.46 & 0.52 & 0.63 & 0.46 & 0.46 & 0.46 & 0.45 \\
\hline $\mathrm{Yb}$ & 3.1 & 3.5 & 4.4 & 3 & 3 & 3 & 3 \\
\hline $\mathrm{Lu}$ & 0.44 & 0.5 & 0.62 & 0.43 & 0.43 & 0.43 & 0.42 \\
\hline
\end{tabular}

CIPW Norm (\%)

\begin{tabular}{|c|c|c|c|c|c|c|c|}
\hline An & 23.01 & 22.87 & 22.76 & 23.62 & 22.60 & 23.70 & 23.15 \\
\hline $\mathrm{Ab}$ & 22.54 & 23.32 & 22.95 & 23.05 & 23.54 & 21.33 & 22.72 \\
\hline Or & 3.36 & 2.92 & 3.13 & 2.95 & 2.84 & 3.30 & 3.20 \\
\hline $\mathrm{Di}$ & 11.81 & 11.64 & 11.90 & 11.44 & 11.31 & 11.63 & 11.84 \\
\hline $\mathrm{Hd}$ & 12.25 & 12.35 & 12.48 & 11.86 & 12.35 & 12.00 & 12.38 \\
\hline Ens & 8.27 & 7.09 & 7.94 & 7.21 & 6.55 & 7.37 & 7.48 \\
\hline Fs & 9.84 & 8.62 & 9.54 & 8.58 & 8.21 & 8.72 & 8.98 \\
\hline Il & 3.81 & 3.89 & 3.81 & 3.89 & 4.05 & 3.90 & 3.84 \\
\hline Mag & 2.68 & 2.75 & 2.70 & 2.72 & 2.83 & 2.80 & 2.71 \\
\hline Fo & 0.87 & 1.77 & 1.01 & 1.84 & 2.23 & 2.10 & 1.40 \\
\hline Fy & 1.14 & 2.37 & 1.34 & 2.41 & 3.08 & 2.73 & 1.85 \\
\hline Total & 99.58 & 99.58 & 99.57 & 99.57 & 99.58 & 99.57 & 99.56 \\
\hline Sample & 008 & 010 & 015 & 016 & 023 & 024 & 026 \\
\hline Level & LF 5-S & LF 7 & LF $1 A$ & LF 2A & BF-NO & LF 8 & BF-N \\
\hline North & $7,803,888$ & $7,804,008$ & $7,804,013$ & $7,803,894$ & $7,803,905$ & $7,803,881$ & $7,804,032$ \\
\hline East & 566,945 & 566,915 & 566,926 & 566,958 & 566,968 & 567,050 & 566,976 \\
\hline \multicolumn{8}{|c|}{ Major elements (wt \%) } \\
\hline $\mathrm{SiO}_{2}$ & 49.58 & 48.15 & 47.63 & 47.29 & 47.64 & 48.1 & 48.66 \\
\hline $\mathrm{TiO}_{2}$ & 2.05 & 1.96 & 1.98 & 1.95 & 1.94 & 1.97 & 1.97 \\
\hline $\mathrm{Al}_{2} \mathrm{O}_{3}$ & 13.54 & 13.09 & 13.20 & 12.67 & 12.52 & 13.05 & 13.19 \\
\hline $\mathrm{Fe}_{2} \mathrm{O}_{3}(\mathrm{t})$ & 14.45 & 13.9 & 14.03 & 13.94 & 14.06 & 13.74 & 14.06 \\
\hline $\mathrm{MnO}$ & 0.21 & 0.20 & 0.21 & 0.19 & 0.18 & 0.20 & 0.20 \\
\hline
\end{tabular}


Table 2. Continuation.

\begin{tabular}{c|c|c|c|c|c|c|c}
\hline Sample & $\mathbf{0 0 8}$ & $\mathbf{0 1 0}$ & $\mathbf{0 1 5}$ & $\mathbf{0 1 6}$ & $\mathbf{0 2 3}$ & $\mathbf{0 2 4}$ & $\mathbf{0 2 6}$ \\
\hline Level & LF 5-S & $\mathbf{L F} \mathbf{7}$ & $\mathbf{L F} \mathbf{~ A}$ & $\mathbf{L F} \mathbf{2 A}$ & $\mathbf{B F - N O}$ & $\mathbf{L F} \mathbf{8}$ & $\mathbf{B F - N}$ \\
\hline $\mathrm{MgO}$ & 6.10 & 5.87 & 5.78 & 5.55 & 5.52 & 5.87 & 5.95 \\
\hline $\mathrm{CaO}$ & 10.32 & 9.97 & 9.91 & 9.69 & 9.83 & 10.08 & 10.16 \\
\hline $\mathrm{Na}_{2} \mathrm{O}$ & 2.73 & 2.54 & 2.68 & 2.56 & 2.61 & 2.61 & 2.66 \\
\hline $\mathrm{K}_{2} \mathrm{O}$ & 0.53 & 0.59 & 0.50 & 0.55 & 0.52 & 0.50 & 0.53 \\
\hline $\mathrm{P}_{2} \mathrm{O}_{5}$ & 0.21 & 0.22 & 0.21 & 0.21 & 0.21 & 0.21 & 0.22 \\
\hline $\mathrm{Cr}_{2} \mathrm{O}_{3}$ & 0.01 & 0.01 & 0.01 & 0.01 & 0.01 & 0.01 & 0.01 \\
\hline $\mathrm{LOI}$ & 0.56 & 0.55 & 0.49 & 0.64 & 0.75 & 0.66 & 0.64 \\
\hline $\mathrm{Total}$ & 100.3 & 97.05 & 96.63 & 95.25 & 95.79 & 96.99 & 98.24 \\
\hline
\end{tabular}

Minor and trace elements (ppm)

\begin{tabular}{|c|c|c|c|c|c|c|c|}
\hline $\mathrm{Zn}$ & 89 & 90 & 82 & 83 & 82 & 86 & 87 \\
\hline $\mathrm{Cu}$ & 237 & 229 & 224 & 226 & 220 & 230 & 226 \\
\hline $\mathrm{Ni}$ & 68 & 64 & 64 & 63 & 62 & 63 & 66 \\
\hline $\mathrm{Ba}$ & 273 & 295 & 270 & 261 & 310 & 286 & 269 \\
\hline Cs & 0.2 & 0.33 & 0.29 & 0.39 & 0.31 & 0.17 & 0.36 \\
\hline $\mathrm{Ga}$ & 20.8 & 20.3 & 20 & 19.7 & 19.9 & 20.5 & 20 \\
\hline $\mathrm{Hf}$ & 3.94 & 3.85 & 5.51 & 3.77 & 4.29 & 3.97 & 3.78 \\
\hline $\mathrm{Nb}$ & - & 12.16 & 17.38 & 11.78 & 15.15 & 12.2 & 12.1 \\
\hline $\mathrm{Rb}$ & 15.2 & 17.3 & 15.1 & 21.1 & 19.3 & 14 & 19.6 \\
\hline Sn & 2.3 & 2.3 & 3.3 & 2.3 & 0.9 & $<0.3$ & 0.8 \\
\hline $\mathrm{Sr}$ & 307 & 298 & 309 & 289 & 296 & 304 & 303 \\
\hline Th & 2.5 & 2.8 & 5.6 & 2.6 & 3.3 & 2.8 & 3 \\
\hline $\mathrm{U}$ & 0.68 & 0.59 & 0.95 & 0.51 & 0.66 & 0.55 & 0.57 \\
\hline V & 436 & 414 & 411 & 397 & 411 & 404 & 421 \\
\hline $\mathrm{Zr}$ & 118 & 113 & 132 & 112 & 115 & 114 & 116 \\
\hline $\mathrm{Y}$ & 29.31 & 28.65 & 28.53 & 28.14 & 28.49 & 28.93 & 29.23 \\
\hline $\mathrm{La}$ & 23.1 & 20.2 & 22.2 & 19.3 & 21.4 & 20.2 & 23.1 \\
\hline $\mathrm{Ce}$ & 43.3 & 41.7 & 41.4 & 40.7 & 41.5 & 41 & 42.2 \\
\hline $\operatorname{Pr}$ & 5.6 & 5.43 & 5.3 & 5.29 & 5.42 & 5.37 & 5.51 \\
\hline $\mathrm{Nd}$ & 22.7 & 22.5 & 21.7 & 21.8 & 22.1 & 21.7 & 22.3 \\
\hline $\mathrm{Sm}$ & 5.5 & 5.3 & 5.3 & 5.4 & 5.3 & 5.1 & 5.3 \\
\hline $\mathrm{Eu}$ & 1.72 & 1.66 & 1.6 & 1.65 & 1.66 & 1.61 & 1.59 \\
\hline $\mathrm{Gd}$ & 5.75 & 5.68 & 5.59 & 5.52 & 5.58 & 5.52 & 5.5 \\
\hline $\mathrm{Tb}$ & 0.9 & 0.89 & 0.89 & 0.86 & 0.88 & 0.89 & 0.89 \\
\hline Dy & 5.72 & 5.61 & 5.58 & 5.42 & 5.47 & 5.48 & 5.5 \\
\hline Ho & 1.13 & 1.12 & 1.11 & 1.09 & 1.11 & 1.12 & 1.08 \\
\hline
\end{tabular}


Table 2. Continuation.

\begin{tabular}{|c|c|c|c|c|c|c|c|}
\hline Sample & 008 & 010 & 015 & 016 & 023 & 024 & 026 \\
\hline Level & LF 5-S & LF 7 & LF 1A & LF 2A & BF-NO & LF 8 & BF-N \\
\hline $\mathrm{Er}$ & 3.23 & 3.16 & 3.08 & 3.03 & 3.09 & 3.13 & 3.12 \\
\hline $\mathrm{Tm}$ & 0.45 & 0.45 & 0.46 & 0.42 & 0.44 & 0.44 & 0.44 \\
\hline $\mathrm{Yb}$ & 3 & 2.9 & 3 & 2.9 & 2.9 & 2.9 & 2.9 \\
\hline $\mathrm{Lu}$ & 0.44 & 0.44 & 0.44 & 0.41 & 0.44 & 0.42 & 0.42 \\
\hline \multicolumn{8}{|c|}{ CIPW Norm (\%) } \\
\hline An & 23.19 & 23.39 & 23.42 & 22.68 & 22.00 & 23.27 & 23.50 \\
\hline $\mathrm{Ab}$ & 23.17 & 22.27 & 23.59 & 22.9 & 23.24 & 22.93 & 22.74 \\
\hline Or & 3.14 & 3.61 & 3.07 & 3.44 & 3.23 & 3.07 & 3.32 \\
\hline $\mathrm{Di}$ & 11.48 & 11.36 & 11.17 & 11.14 & 11.52 & 11.76 & 11.70 \\
\hline $\mathrm{Hd}$ & 11.95 & 11.83 & 11.92 & 12.32 & 12.95 & 12.07 & 11.75 \\
\hline Ens & 6.87 & 7.81 & 6.21 & 7.68 & 7.56 & 7.49 & 7.85 \\
\hline Fs & 8.20 & 9.33 & 7.60 & 9.75 & 9.75 & 8.81 & 9.04 \\
\hline Il & 3.90 & 3.86 & 3.91 & 3.91 & 3.88 & 3.88 & 3.77 \\
\hline Mag & 2.74 & 2.72 & 2.76 & 2.79 & 2.80 & 2.70 & 2.66 \\
\hline Fo & 2.13 & 1.45 & 2.51 & 1.24 & 1.10 & 1.57 & 1.44 \\
\hline Fy & 2.80 & 1.91 & 3.39 & 1.73 & 1.56 & 2.03 & 1.82 \\
\hline Total & 99.58 & 99.56 & 99.56 & 99.58 & 99.59 & 99.57 & 99.58 \\
\hline
\end{tabular}

LF: lava flow; BF: basal flow; An: anorthite; Ab: albite; Or: orthoclase; Di: diopside; Hd: hedenbergite; Ens: enstatita; Fs: ferrossilite; Il: ilmenita; Mag: magnetite; Fo: forsterite; Fy: fayalite.

The HFSE and REE arrangement (Thompson et al. 1984) normalized to the mid-ocean ridge basalt (MORB) (Sun \& McDonough 1989) can demonstrate features of the original magma (Pearce 2008). The significant negative $\mathrm{Nb}$ anomaly (Fig. 10C) is a characteristic chemical signature for some continental flood basalt (e.g., Arndt \& Christensen 1992, Pik et al. 1999), reflecting the source composition and melt conditions (Turner \& Hawkesworth 1995). The PEMP model for melt generation suggests the partial fusion of peridotite on the subcontinental lithospheric mantle (SCLM), chemically modified due to previous subduction processes which generated the negative $\mathrm{Nb}$ anomaly (Turner et al. 1996, Reichow et al. 2005, Wang et al. 2008).

The additional normalization of the incompatible elements to $\mathrm{Ti}=1$, showed in Figure 10D, assists the visualization of the effects of crustal contamination (segment A), source composition and degree of partial melting (segment B) and depth of melt generation (segment C) (Pearce 2008). Although the $\mathrm{Th}_{\mathrm{N}}$ shows high values, as aforementioned, the other proxies do not show crustal contamination. The anomalous high $\mathrm{Th}, \mathrm{U}, \mathrm{Zr}$ and $\mathrm{Hf}$ concentrations draw our attention and should be observed in future works on rocks in that area. Although some laboratory contamination cannot be completely ruled out, the rigor with which the samples preparation was made does not allow us to impute the anomalies before the analysis process. The segments $\mathrm{B}$ and $\mathrm{C}$ reveal the magma origin in an enriched source with low degree of partial melting and at high depths. The behavior of average composition of Paranapanema samples that did not suffer crustal contamination (based on initial $\mathrm{Sr}$ isotope ratios $<0.7060$ ) (Marques et al. 2017) is very similar to this work samples.

However, the average composition of the Água Vermelha basalts shows some differences from the average composition of other Paranapanema rocks, with slightly lower contents of $\mathrm{SiO}_{2}, \mathrm{TiO}_{2}, \mathrm{Fe}_{2} \mathrm{O}_{3}, \mathrm{~K}_{2} \mathrm{O}$ and $\mathrm{P}_{2} \mathrm{O}_{5}$ and higher contents of $\mathrm{MgO}, \mathrm{CaO}$ and $\mathrm{Na}_{2} \mathrm{O}$ compared to the samples from Rocha-Júnior et al. (2013), Machado et al. (2007) and Pinto \& Hartmann (2011) (Fig. 11). 


\section{MINERAL CHEMISTRY}

The composition and variation of each mineral phase were obtained for the samples $003 \mathrm{~b}$ and 022 (lava flows), and the samples 006, 023 and 027 (basal flows) are shown and discussed in this topic. The core and rim of the crystals of plagioclase and clinopyroxene and microlites of plagioclase, pyroxene and titanomagnetite were analyzed.

\section{Plagioclase}

The plagioclases were classified according to the Or-Ab-An diagram (Deer et al. 2003) (Fig. 12). Plagioclase microlites from both lava and basal flows are composed of labradorite, with anorthite (An), albite (Ab) and orthoclase (Or) contents between $\mathrm{An}_{59} \mathrm{Ab}_{39} \mathrm{Or}_{2}$ and
$\mathrm{An}_{67} \mathrm{Ab}_{32} \mathrm{Or}_{1}$. The phenocrysts from the lava and basal flows show a very weak compositional zoning (Tab. 3). The chemical formula for the phenocrysts of the lava flow can be summarized as $\mathrm{An}_{80} \mathrm{Ab}_{19} \mathrm{Or}_{1}$ and it is characterized as bytownite. There are two distinct groups of phenocrysts on the basal flow, with a slightly different composition from rim to core, being the first group characterized as bytownite $\left(\mathrm{An}_{81} \mathrm{Ab}_{18} \mathrm{Or}_{1}\right.$ to $\left.\mathrm{An}_{70} \mathrm{Ab}_{29} \mathrm{Or}_{1}\right)$ and the second one as labradorite $\left(\mathrm{An}_{69} \mathrm{Ab}_{30} \mathrm{Or}_{1}\right.$ to $\left.\mathrm{An}_{64} \mathrm{Ab}_{34} \mathrm{Or}_{2}\right)$. Table 3 summarizes the data of plagioclases analysis.

\section{Pyroxene}

The clinopyroxene found in the samples is classified as augite in the Wo-En-Fs diagram of Morimoto (1988) (Fig. 13). The main features of the crystals are presented in Table 4.
A

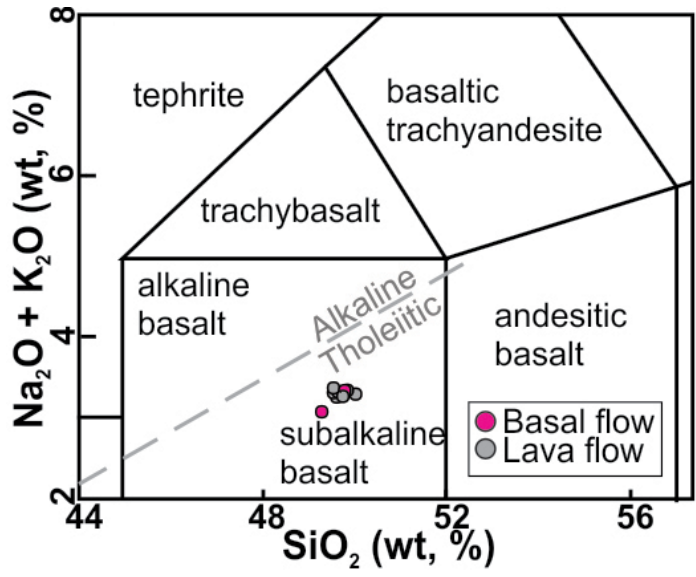

C

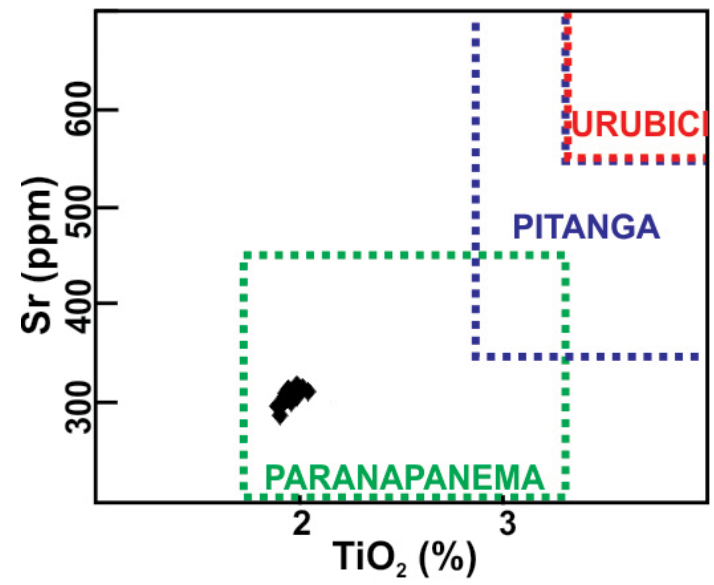

B
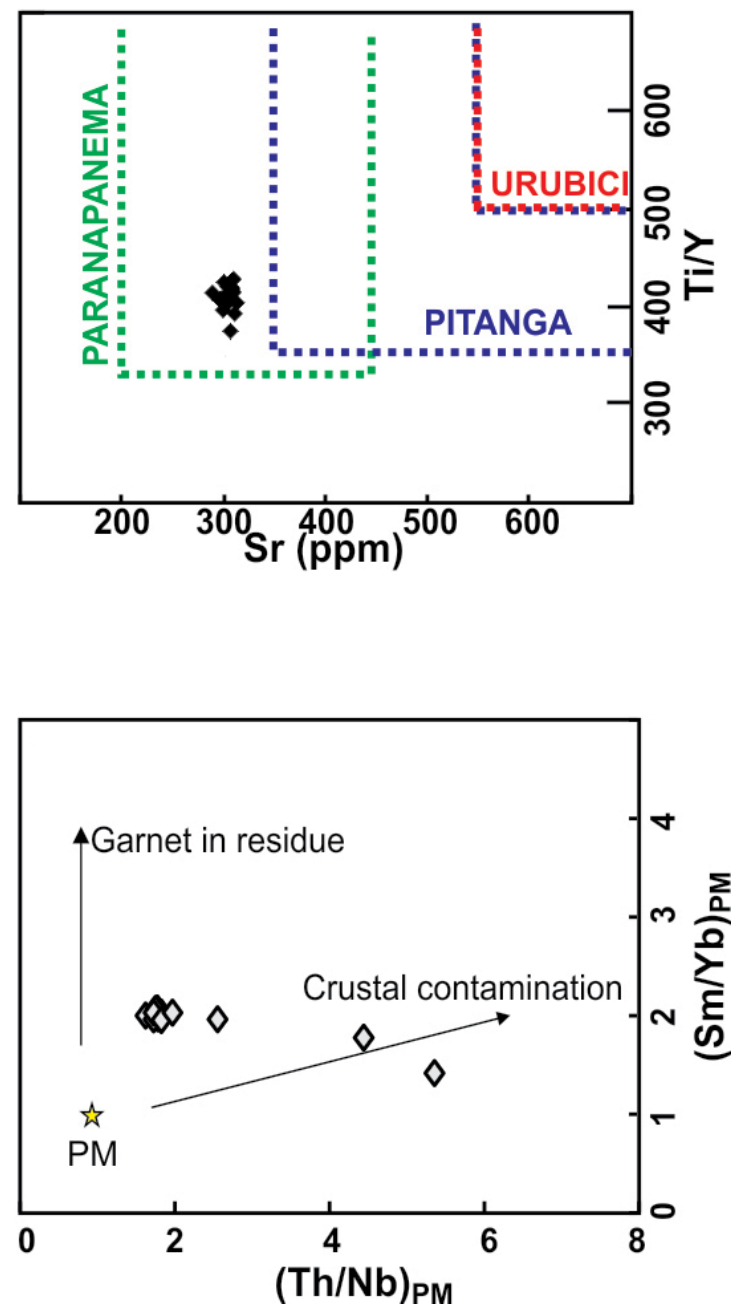

Figure 8. (A) Total alkalis/silica (TAS) diagram (Le Maitre 2002) with the line that divides the alkaline and tholeiitic rocks fields (MacDonald \& Katsura 1964); (B and C) diagrams for discrimination of high titanium magma-types of the Paraná-Etendeka Province (Peate et al. 1992, Machado et al. 2007); (D) (Th/Nb) ${ }_{\mathrm{PM}}$ vs. (Sm/Yb) PM $_{\mathrm{P}}$ diagram for crustal contamination (Wang et al. 2007). 


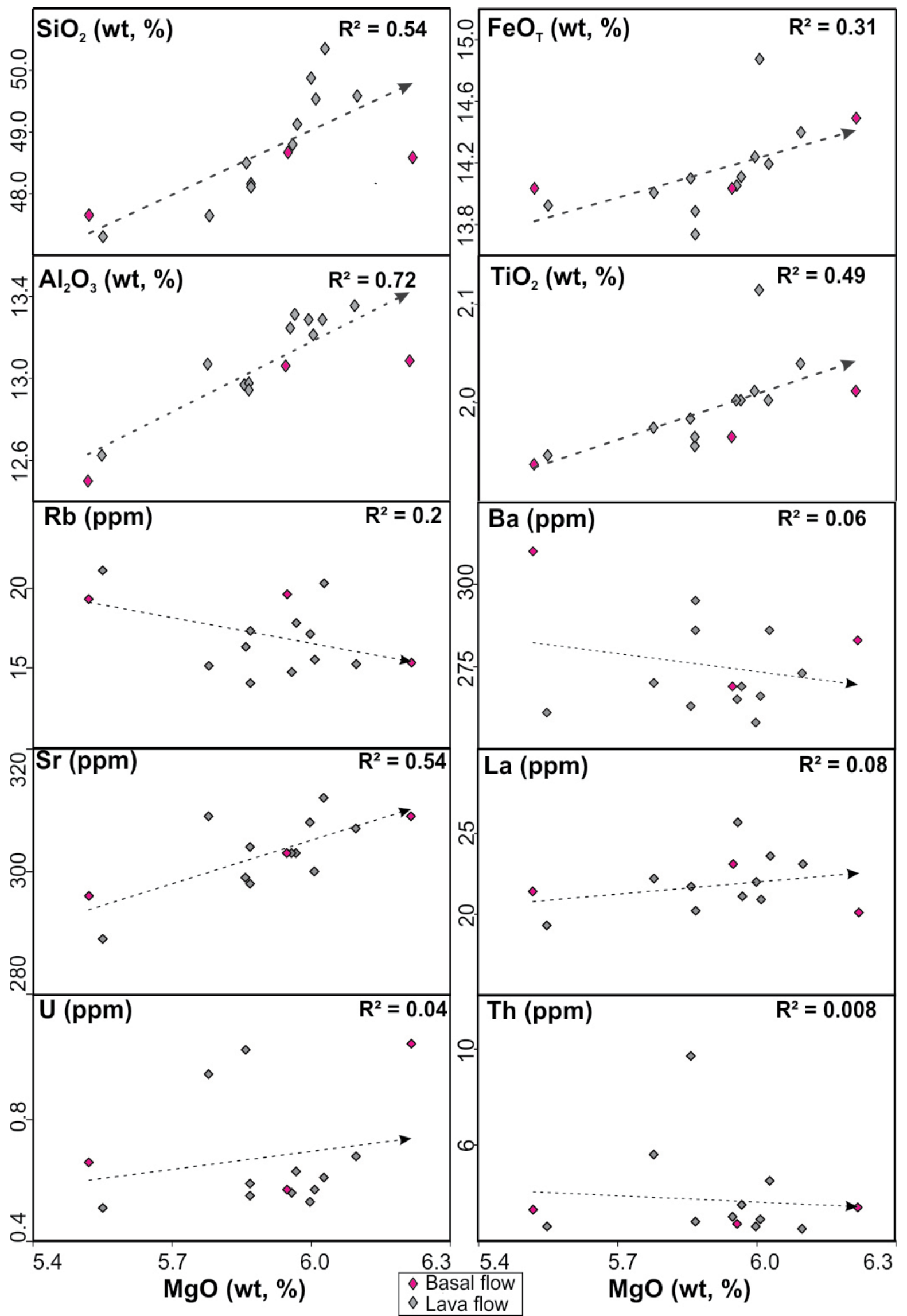

Figure 9. Bivariant diagrams of major $\left(\mathrm{SiO}_{2}, \mathrm{FeO}_{\mathrm{T}}, \mathrm{Al}_{2} \mathrm{O}_{3}\right.$ and $\left.\mathrm{TiO}_{2}\right)$ and trace $(\mathrm{Rb}, \mathrm{Ba}, \mathrm{Sr}, \mathrm{La}, \mathrm{U}$ and $\mathrm{Th})$ elements $v$ s. $\mathrm{MgO}$. 


\section{Titanomagnetite (ulvöspinel)}

The oxide analyses were plotted on the ternary $\mathrm{FeO}$ vs. $\mathrm{TiO}_{2}$ vs. $\mathrm{Fe}_{2} \mathrm{O}_{3}$ diagram (Akimoto \& Katsura 1959). Since the microanalysis considered only $\mathrm{FeO}$, the estimates of $\mathrm{FeO}$ and $\mathrm{Fe}_{2} \mathrm{O}_{3}$ were made as described in the Materials and Methods section. This diagram shows the major solid solution series magnetite-ulvöspinel, hematite-ilmenite and ferropseudobrookite-pseudobrookite (Fig. 14A). The skeletal oxides (Fig. 14B) analyzed for both lava and basal flows plot on the solid solution series of magnetite-ulvöspinel (titanomagnetite), and are close to the ulvöspinel end-member. Table 5 summarizes the data for the titanomagnetite analysis.

\section{DISCUSSION}

The petrography and whole-rock analyses show that the basalts of Água Vermelha have a subalkaline and

A

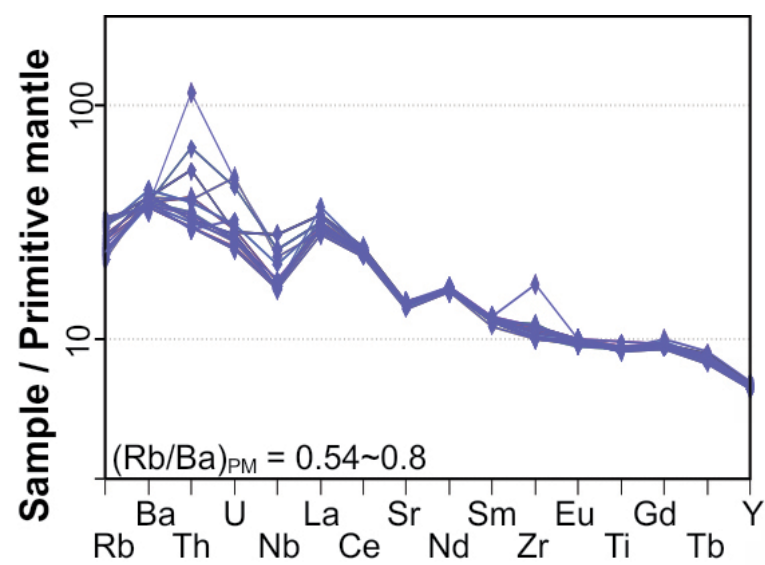

C

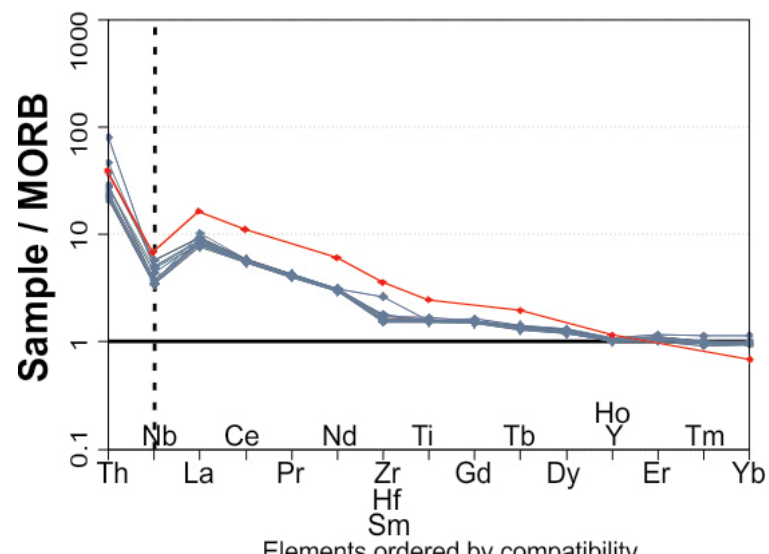

tholeiitic signature due to the alkali and silica contents, as well as the presence of normative olivine and enstatite (Machado et al. 2007). The $\mathrm{MgO}$ content of BRS samples increases with the enrichment of silica, different from the

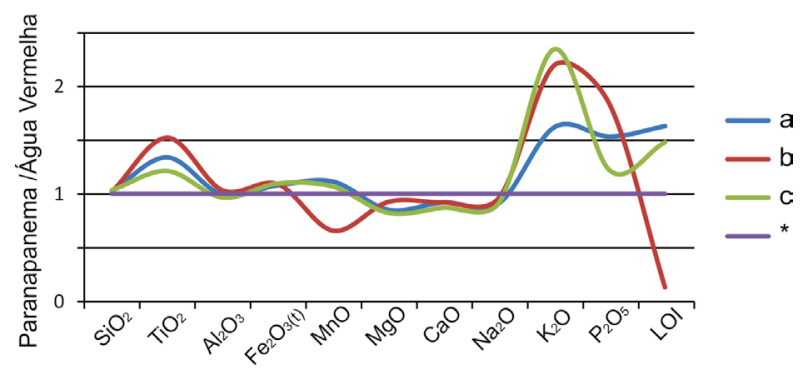

Figure 11. Comparative chart between Paranapanema average compositions normalized to the average composition samples from this work $\left({ }^{*}\right)$. (A) RochaJúnior et al. (2013), 10 samples; (B) Machado et al. (2007), two samples; (C) Pinto \& Hartmann (2011), 14 samples.

B

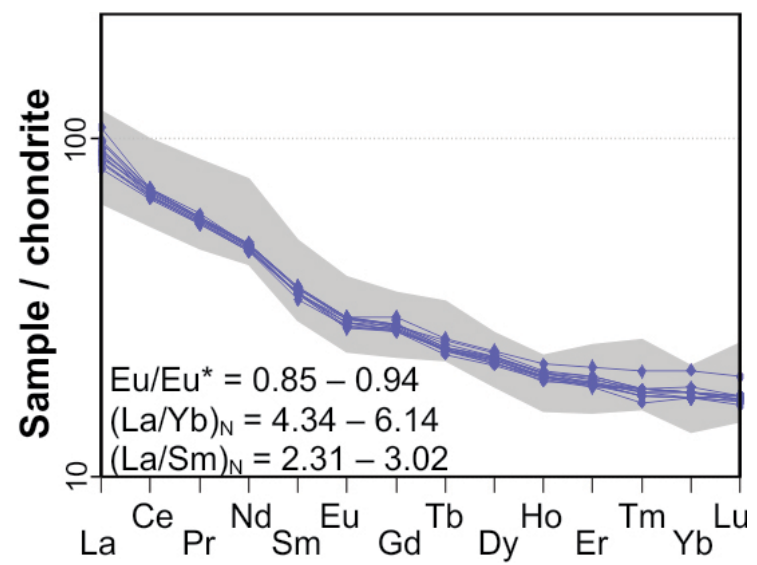

D

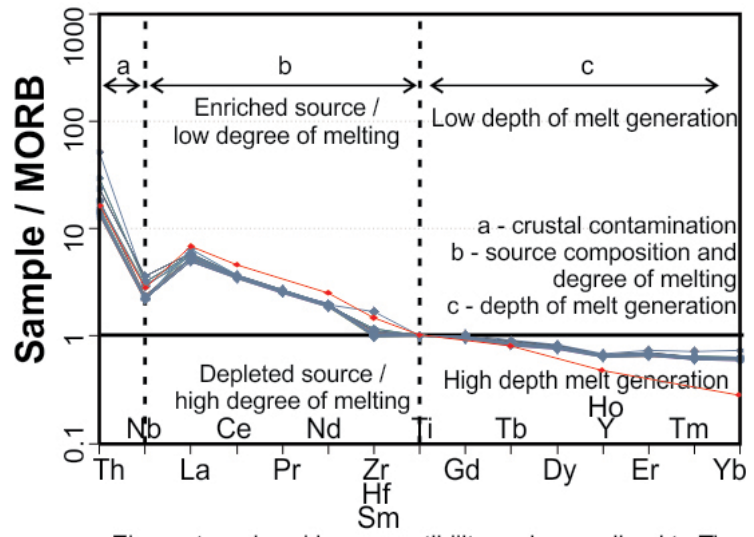

Elements ordered by compatibility and normalized to $\mathrm{Ti}$

Figure 10. (A) Minor and trace elements normalized to the primitive mantle (Sun \& McDonough 1989); (B) rare earth elements (REE) normalized to chondrite (Sun \& McDonough 1989). Gray field represents the Paranapanema type basalts, with data from Pinto \& Hartmann (2011). (C and D) Incompatible elements normalized to midocean ridge basalt (MORB) (Sun \& McDonough 1989) and to $\mathrm{Ti}=1$. (Pearce 2008) The red line represents the average sample composition of Paranapanema samples (Rocha-Júnior et al. 2013) that did not suffer crustal contamination (based on initial $\mathrm{Sr}$ isotope ratios $<0.7060$ ). 

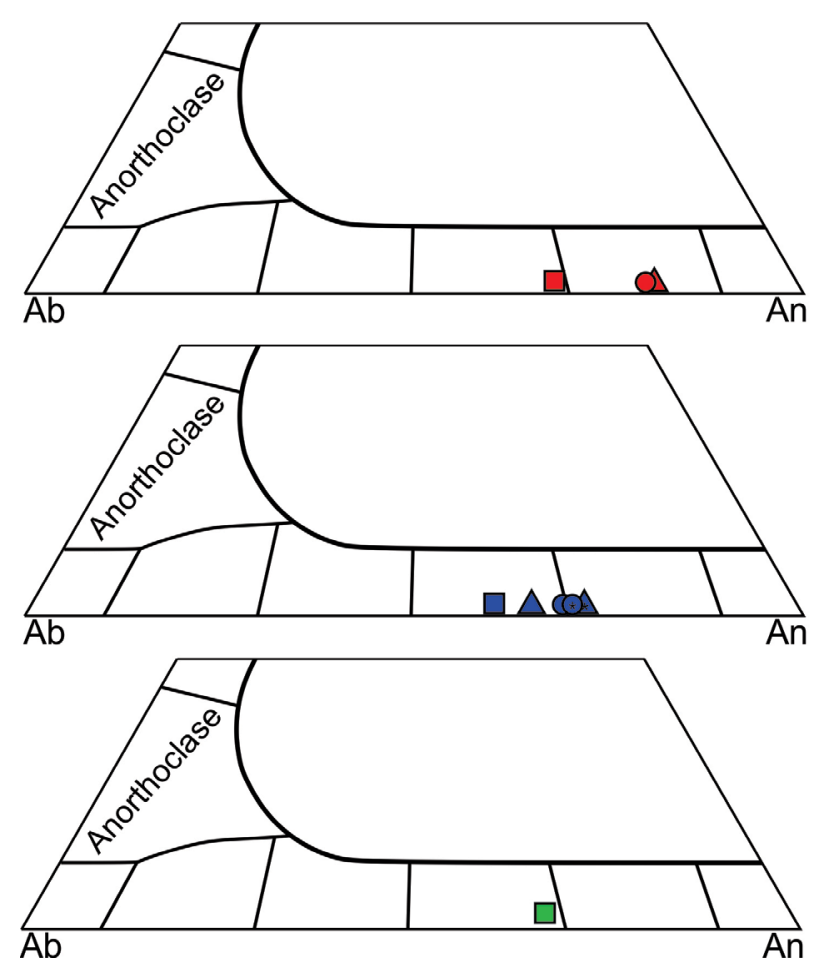

Circle: core; triangle: rim; square: microlite.

Figure 12. Ternary diagram plot (Or-Ab-An) (Deer et al. 2003) for plagioclases from samples of the ring structure E6.

Table 3. Summary of plagioclase data from samples of the ring structure E6.

\begin{tabular}{|c|c|c|c|c|c|}
\hline Sample & Cristal & $\% O r$ & $\% A b$ & $\%$ An & Mineral \\
\hline \multicolumn{6}{|c|}{ Lava flow } \\
\hline \multirow{3}{*}{ 003b } & Microlite & 1 & 31 & 67 & Labradorite \\
\hline & Phenocryst (core) & 1 & 20 & 80 & Bytownite \\
\hline & Phenocryst (rim) & 1 & 20 & 80 & Bytownite \\
\hline 22 & Microlite & 1 & 33 & 66 & Labradorite \\
\hline \multicolumn{6}{|c|}{ Basal flow } \\
\hline \multirow{5}{*}{6} & Microlite & 2 & 39 & 59 & Labradorite \\
\hline & Phenocryst (core) & 1 & 30 & 69 & Labradorite \\
\hline & Phenocryst (rim) & 1 & 34 & 64 & Labradorite \\
\hline & Phenocryst (core) & 1 & 30 & 69 & Bytownite \\
\hline & Phenocryst (rim) & 1 & 29 & 70 & Bytownite \\
\hline \multirow{5}{*}{27} & Microlite & 1 & 32 & 67 & Labradorite \\
\hline & Phenocryst (core) & 1 & 18 & 81 & Bytownite \\
\hline & Phenocryst (rim) & 1 & 19 & 80 & Bytownite \\
\hline & Phenocryst (core) & 1 & 33 & 66 & Labradorite \\
\hline & Phenocryst (rim) & 1 & 31 & 68 & Labradorite \\
\hline 23 & Microlite & 1 & 32 & 67 & Labradorite \\
\hline
\end{tabular}

Or: orthoclase; Ab: albite; An: anorthite.
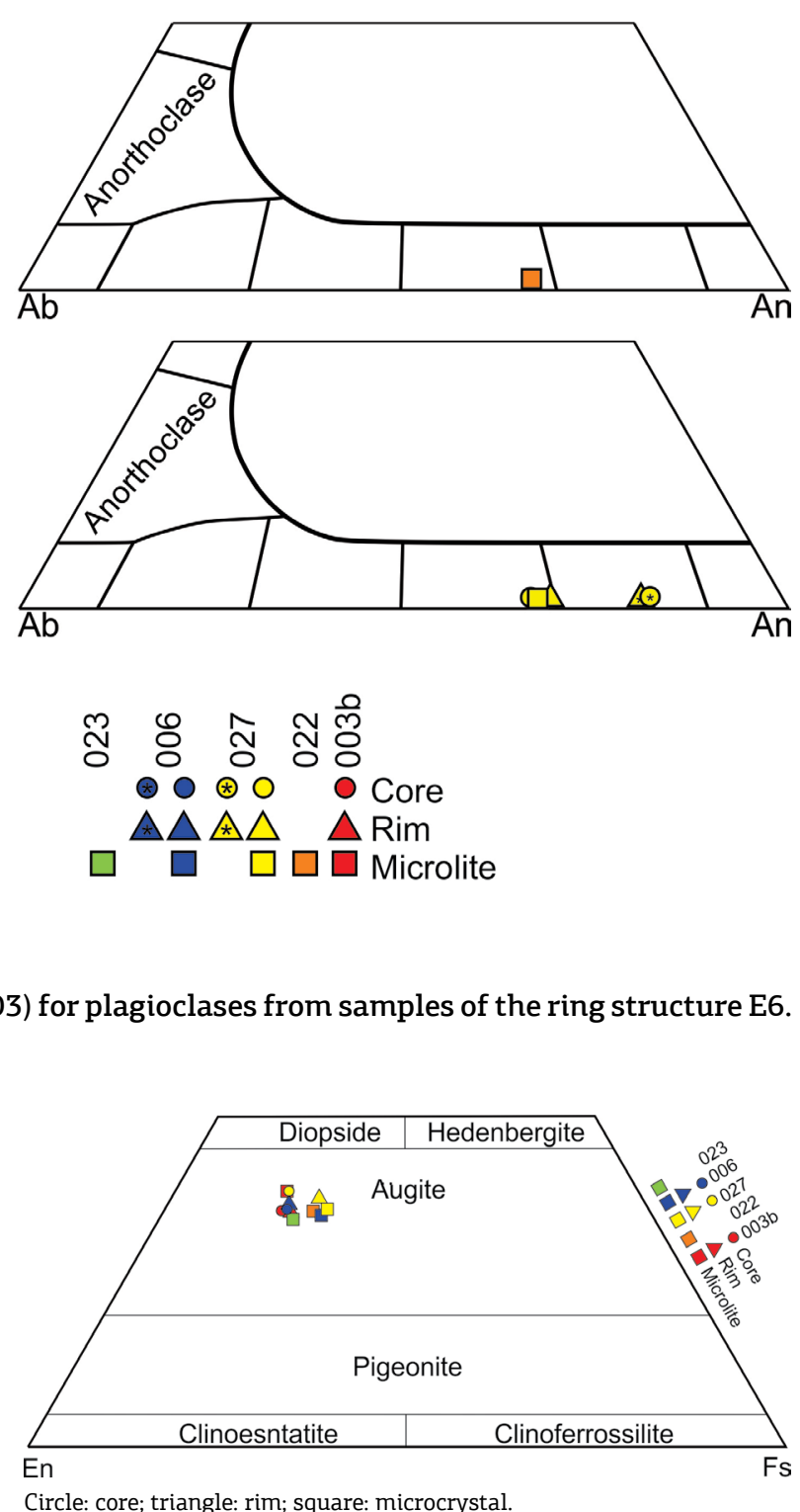

Figure 13. Ternary diagram plot (Wo-En-Fs) (Morimoto 1988) for clynopyroxenes from ring structure E6.

other samples of Paranapanema-type basalts, while the content of $\mathrm{TiO}_{2}, \mathrm{~K}_{2} \mathrm{O}$ and $\mathrm{P}_{2} \mathrm{O}_{5}$ stay constant with the increase of silica and it is significantly lower than typical Paranapanema. Also, they present enrichment of $\mathrm{Al}_{2} \mathrm{O}_{3}$ $\mathrm{MgO}, \mathrm{CaO}$ and $\mathrm{Na}_{2} \mathrm{O}$ related to other Paranapanema samples (Rocha-Júnior et al. 2013, Machado et al. 2007, Pinto \& Hartmann 2011) (Fig. 15).

The mineral chemistry allows determining that the plagioclase of both lava and basal flows has a composition ranging between bytownite and labradorite. The plagioclase with slightly Ca-richer rim than the core suggests the possibility of new magma injections on the chambers. The pyroxenes of the basal and lava flow are classified as augite. 
The Eu anomaly is absent when the samples are normalized to the primitive mantle and it is negative and subtle when normalized to the chondrite. This slight anomaly probably represents minor plagioclase fractionation. On the other hand, the Sr anomaly can be related to plagioclase fractionation, which is consistent with the presence of plagioclase phenocrysts and a glomeroporphyritic texture (Pinto \& Hartmann 2011).

The increase of $\mathrm{CaO}$ and $\mathrm{MgO}$ related to the $\mathrm{SiO}_{2}$ content cannot be explained by the fractional crystallization alone. Instead, the presence of the lava lake on the Água Vermelha BRS, with events of effusion and episodes of explosion (e.g., spatters), indicates new magma pulses on the shallow chambers, modifying the oxide contents. The $\mathrm{CaO}$ and $\mathrm{MgO}$ distinct trend behavior between the
BRS samples and other Paranapanema types could be explained by the refilling of these chambers by more than one parental magma. O'Hara (1977) describes a model in which the magma in a high-level chamber suffers continuous fractional crystallization and it is periodically fed

Table 5. Summary for titanomagnetite data from samples of the ring structure E6.

\begin{tabular}{l|c|c|c|c}
\hline & Samples & $\% \mathrm{FeO}$ & $\%_{\mathrm{Fe}_{2} \mathbf{O}_{3}}$ & $\% \mathbf{T i O}_{2}$ \\
\hline Lava Flow & 003b & 67,56 & 7,52 & 24,91 \\
\hline \multirow{2}{*}{ Basal Flow } & 006 & 64,49 & 7,18 & 28,33 \\
\cline { 2 - 5 } & 023 & 67,01 & 7,46 & 25,53 \\
\hline
\end{tabular}

Table 4. Summary of pyroxene data from samples of the ring structure E6.

\begin{tabular}{|c|c|c|c|c|c|}
\hline Sample & Crystal type & Chemical formula & $\%$ Fs & $\%$ En & \%Wo \\
\hline \multicolumn{6}{|c|}{ Lava flow } \\
\hline \multirow{3}{*}{ 003b } & Microlite & $\left(\mathrm{Ca}_{0,76} \mathrm{Na}_{0,01}\right)\left(\mathrm{Mg}_{0,90} \mathrm{Fe}_{0,28} \mathrm{Ti}_{0,02} \mathrm{Al}_{0,02}\right)\left(\mathrm{Si}_{1,93} \mathrm{Al}_{0,07}\right) \mathrm{O}_{6}$ & 14,34 & 46,59 & 39,07 \\
\hline & Phenocryst (core) & $\left(\mathrm{Ca}_{0,70} \mathrm{Na}_{0,01}\right)\left(\mathrm{Mg}_{0,94} \mathrm{Fe}_{0,29} \mathrm{Ti}_{0,02} \mathrm{Al}_{0,01}\right)\left(\mathrm{Si}_{1,89} \mathrm{Al}_{0,11}\right) \mathrm{O}_{6}$ & 15,23 & 48,81 & 35,96 \\
\hline & Phenocryst (rim) & $\left(\mathrm{Ca}_{0,69} \mathrm{Na}_{0,01}\right)\left(\mathrm{Mg}_{0,93} \mathrm{Fe}_{0,32} \mathrm{Ti}_{0,02} \mathrm{Al}_{0,08}\right)\left(\mathrm{Si}_{1,93} \mathrm{Al}_{0,07}\right) \mathrm{O}_{6}$ & 16,47 & 47,68 & 35,85 \\
\hline 022 & Microlite & $\left(\mathrm{Ca}_{0,70} \mathrm{Na}_{0,01}\right)\left(\mathrm{Mg}_{0,87} \mathrm{Fe}_{0,38} \mathrm{Ti}_{0,03} \mathrm{Al}_{0,02}\right)\left(\mathrm{S}_{\mathrm{i} 1,92} \mathrm{Al}_{0,08}\right) \mathrm{O}_{6}$ & 19,34 & 44,80 & 35,86 \\
\hline \multicolumn{6}{|c|}{ Basal flow } \\
\hline \multirow{3}{*}{006} & Microlite & $\left(\mathrm{Ca}_{0,70} \mathrm{Na}_{0,01}\right)\left(\mathrm{Mg}_{0,86} \mathrm{Fe}_{0,41} \mathrm{Ti}_{0,02} \mathrm{Al}_{0,01}\right)\left(\mathrm{Si}_{1,92} \mathrm{Al}_{0,08}\right) \mathrm{O}_{6}$ & 20,76 & 43,75 & 35,49 \\
\hline & Phenocryst (core) & $\left(\mathrm{Ca}_{0,77} \mathrm{Na}_{0,01}\right)\left(\mathrm{Mg}_{0,93} \mathrm{Fe}_{0,29} \mathrm{Ti}_{0,02}\right)\left(\mathrm{Si}_{1,9} \mathrm{Al}_{0,1}\right) \mathrm{O}_{6}$ & 14,53 & 46,34 & 39,14 \\
\hline & Phenocryst (rim) & $\left(\mathrm{Ca}_{0,75} \mathrm{Na}_{0,01}\right)\left(\mathrm{Mg}_{0,85} \mathrm{Fe}_{0,37} \mathrm{Ti}_{0,02}\right)\left(\mathrm{Si}_{1,91} \mathrm{Al}_{0,09}\right) \mathrm{O}_{6}$ & 19,03 & 42,96 & 38,01 \\
\hline \multirow{3}{*}{027} & Microlite & $\left(\mathrm{Ca}_{0,70} \mathrm{Na}_{0,01}\right)\left(\mathrm{Mg}_{0,82} \mathrm{Fe}_{0,41} \mathrm{Ti}_{0,03} \mathrm{Al}_{0,03}\right)\left(\mathrm{Si}_{1,92} \mathrm{Al}_{0,08}\right) \mathrm{O}_{6}$ & 21,10 & 42,48 & 36,42 \\
\hline & Phenocryst (core) & $\left(\mathrm{Ca}_{0,69} \mathrm{Na}_{0,01}\right)\left(\mathrm{Mg}_{0,91} \mathrm{Fe}_{0,30} \mathrm{Ti}_{0,02} \mathrm{Al}_{0,04}\right)\left(\mathrm{Si}_{1,92} \mathrm{Al}_{0,08}\right) \mathrm{O}_{6}$ & 15,74 & 47,91 & 36,35 \\
\hline & Phenocryst (rim) & $\left(\mathrm{Ca}_{0,75} \mathrm{Na}_{0,02}\right)\left(\mathrm{Mg}_{0,88} \mathrm{Fe}_{0,28} \mathrm{Ti}_{0,02} \mathrm{Al}_{0,03}\right)\left(\mathrm{Si}_{1,92} \mathrm{Al}_{0,08}\right) \mathrm{O}_{6}$ & 15,65 & 47,37 & 36,98 \\
\hline 023 & Microlite & $\left(\mathrm{Ca}_{0,68} \mathrm{Na}_{0,01}\right)\left(\mathrm{Mg}_{0,93} \mathrm{Fe}_{0,33} \mathrm{Ti}_{0,02} \mathrm{Al}_{0,03}\right)\left(\mathrm{Si}_{1,91} \mathrm{Al}_{0,09}\right) \mathrm{O}_{6}$ & 17,30 & 48,00 & 34,70 \\
\hline
\end{tabular}

Fs: ferrossilite; En: enstatite; Wo: wollastonite.
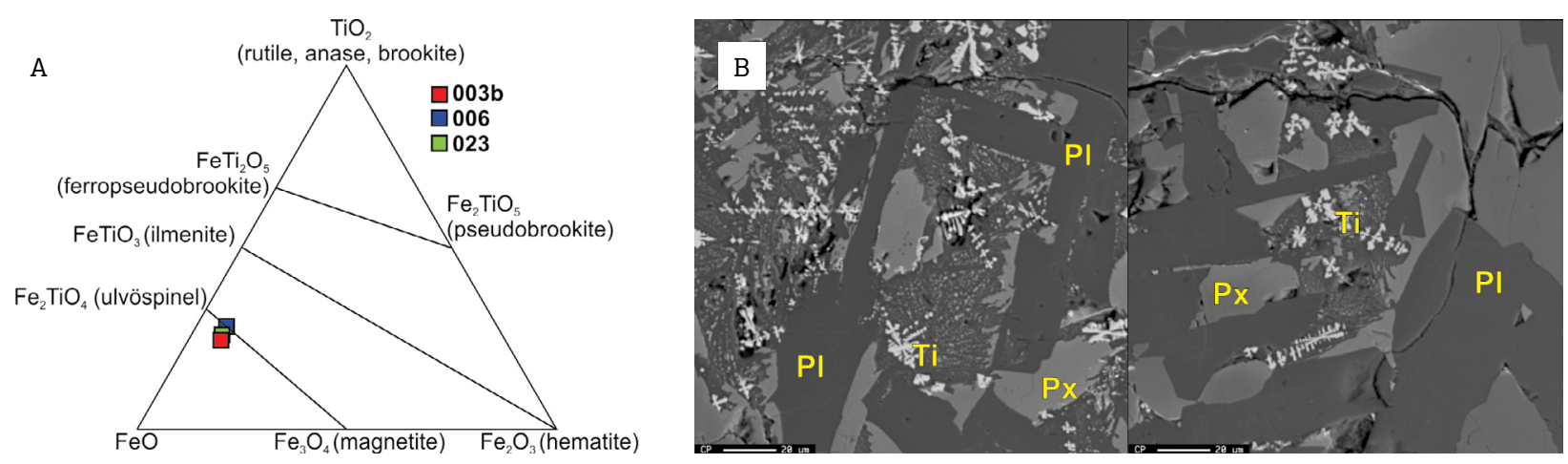

Figure 14. (A) Ternary $\mathrm{FeO}$ vs. $\mathrm{TiO}_{2}$ vs. $\mathrm{Fe}_{2} \mathrm{O}_{3}$ diagram (Akimoto \& Katsura 1959); (B) backscattering electron image, showing titanomagnetite $(\mathrm{Ti})$, plagioclase $(\mathrm{Pl})$ and pyroxene $(\mathrm{Px})$ from a basalt samples of the ring structure E6. 
with new batch from the deep parental magma and, in its model, this influx displaces a portion of the residual liquid from the chamber as a lava flow. The rest of the previous magma mixes with the new batch, and the fractionation process continues to occur. Also, a system that undergoes episodic recharge and eruption can develop distinctly different geochemical characteristics (Spera \& Bohrson 2004).

The analysis of the diagram proposed by Pearce (2008) suggests that the magma was originated in a high depth enriched source, with a low degree of melting. Marques et al. (1999) describe a garnet peridotite as a likely source for the basalts of the Paraná basin, as well as low partial melting of the HTi basalt sources, which is corroborated by the interpretations of the BRS evolution so far. The higher enrichment of LREE related to HREE, the LILE enrichment and the strong negative $\mathrm{Nb}$ anomalies are the main evidences of the involvement of metasomatized components (Rocha-Júnior et al. 2013). According to those authors, the mantle was enriched in fluids and/or magma related to subduction processes during the Neoproterozoic, which hybridize the mantle peridotite with recycled components.
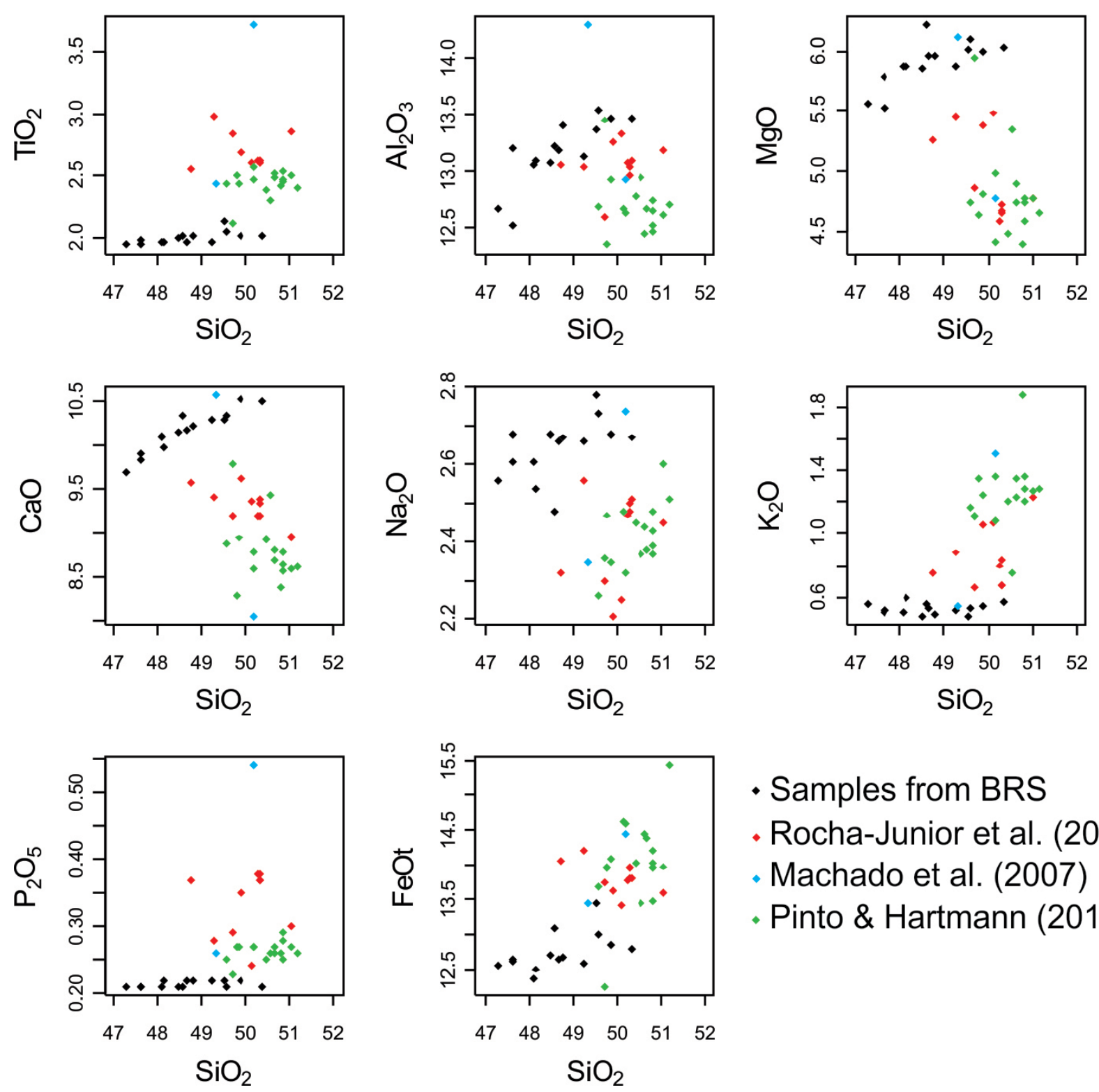

- Samples from BRS

- Rocha-Junior et al. (2013)

- Machado et al. (2007)

- Pinto \& Hartmann (2011)

Figure 15. Harker diagram of $\mathrm{SiO}_{2}$ vs. major elements for samples of the ring structure E6 (basaltic ring structure-BRS) as compared to other Paranapanema-types samples. 


\section{CONCLUSION}

The rocks of the basaltic ring structure from Água Vermelha belong to the Paranapanema magma-type of the high titanium group of basalts from the Serra Geral Formation, but they show some slight differences from other samples of the same magma-type in other places. The whole rock analyses show a subalkaline and tholeiitic signature.

Using the mineral chemistry, it was possible to characterize the plagioclase, pyroxene and oxides of the samples analyzed. The plagioclase has a composition between bytownite and labradorite for both lava and basal flows. The clinopyroxenes are strictly augite. The oxides are characterized as titanomagnetite (ulvöspinel).

Through the whole rock analyses of the lava and basal flows, we could determine that the magma source has a high depth and low degree of partial melting. The magma on the shallow magmatic chamber suffered fractional crystallization and new magma injections, which were responsible for the effusion of samples of the already differentiated magma. The remaining differentiated liquid mixed with the new batches during the evolution of the structure.

The singularities present in Água Vermelha — such as the presence of basaltic ring structures, unusual in PEMP — show the necessity of deeper studies at the region. Thus, geochemical analyses, as well as geological mapping and stratigraphic study, are important to progress on the geological comprehension of the PEMP.

\section{ACKNOWLEDGMENTS}

This work was supported by the Triângulo Mineiro mapping project (funded by Companhia de Desenvolvimento Econômico de Minas Gerais - CODEMIG, in collaboration with CPMTC-UFMG), the Foundation of Support to the Research of the State of Sáo Paulo (Fundação de Amparo à Pesquisa do Estado de São Paulo - FAPESP) (Project 2011/10508-6), and the National Council for Scientific and Technological Development (Conselho Nacional de Desenvolvimento Científico e Tecnológico CNPq). Fabrício de Andrade Caxito, Antônio Carlos Pedrosa-Soares and Glaucia Nascimento Queiroga are fellows of the CNPq and acknowledge the support received. The original manuscript was greatly improved after comments and suggestions of Leila Marques and an anonymous reviewer.

\section{REFERENCES}

Akimoto S.I., Katsura T. 1959. Magneto-chemical study of the generalized titanomagnetite in volcanic rocks. Journal of Geomagnetism and Geoelectricity, 10(3), 69-90. https://doi.org/10.5636/jgg.10.69

Almeida F.F.M. 1986. Distribuição regional e relações tectônicas do magmatismo pós-paleozóico no Brasil. Revista Brasileira de Geociências, 16(4), 325-349.

Araújo J.S. 1982. Estruturas Circulares de Água Vermelha. Master Dissertation, Instituto de Geociências, Universidade de São Paulo, São Paulo, 79 p.

Araújo J.S., Björnberg A.J.S., Silva R.F., Soares L. 1977. A Complex Structure in Basaltic Lava Flows at Água Vermelha Dam - SP Brasil. In: The Geotechnics of Structurally Complex Formations. Proceedings... Capri.

Araújo J.S., Hasui Y. 1985. Estruturas Vulcânicas em Basaltos no Vale do Rio Grande, São Paulo/Minas Gerais. In: Simpósio Regional de Geologia, 5, São Paulo. Atas... Sociedade Brasileira de Geologia, v. 1, p. 289-300.

Arndt N.T., Christensen U. 1992. The role of lithospheric mantle in continental flood volcanism: thermal and geochemical constraints. Journal of Geophysical Research: Solid Earth, 97(B7), 10967-10981.

Bellieni G., Comin-Chiaramonti P., Marques L.S., Melfi A.J., Piccirillo E.M., Nardy A.J.R., Roisenberg A. 1984. High-and low-TiO 2 flood basalts from the Paraná plateau (Brazil): petrology and geochemical aspects bearing on their mantle origin. Neues Jahrbuch für Mineralogie Abhandlungen, 150:273-306.

Burr D.M., Bruno B.C., Lanagan P.D., Glaze L.S., Jaeger W.L., Soare R.J., Tseung J-M.W.B, Skinner Jr. J.A., Baloga S.M. 2009. Mesoscale raised rim depressions (MRRDs) on Earth: A review of the characteristics, processes, and spatial distributions of analogs for Mars. Planetary and Space Science, 57(5-6):579-596. https://doi.org/10.1016/j.pss.2008.11.011

Cross W., Iddings J.P., Pirsson L.V., Washington L.V. 1903. Quantitative Classification of Igneous Rocks. Chicago: University of Chicago Press.

Deckart K., Féraud G., Marques L.S., Bertrand H. 1998. New time constraints on dyke swarms related to the Paraná-Etendeka magmatic province, and subsequent South Atlantic opening, southeastern Brazil. Journal of Volcanology and Geothermal Research, 80(1-2):67-83. https://doi.org/10.1016/S0377-0273(97)00038-3

Deer W.A., Howie R.A., Zussman J. 2003. An Introduction to the Rock-Forming Minerals. New York, Longman Scientific and Technical.

Frank H.T., Gomes M.E.B., Formoso M.L.L. 2009. Review of the areal extent and the volume of the Serra Geral Formation, Paraná Basin, South America. Pesquisas em Geociências, 36(1):49-57.

Gill R. 2010. Igneous rocks and processes: a practical guide. New Jersey, Wiley-Blackwell.

Jaeger W.L., Keszthelyi L.P., Burr D.M., Emery J.P., Baker V.R., McEwen A.S., Miyamoto H. 2005. Basaltic ring structures as an analog for ring features in Athabasca Valles, Mars. In: Annual Lunar and Planetary Science Conference, 36. Proceedings... v. 36.

Janasi V.A., de Freitas V.A., Heaman L.H. 2011. The onset of flood basalt volcanism, Northern Paraná Basin, Brazil: a precise U-Pb baddeleyite/ zircon age for a Chapecó-type dacite. Earth and Planetary Science Letters, $302(1-2): 147-153 . \quad$ https://doi.org/10.1016/j. epsl.2010.12.005 
Johannsen A. 1931. A descriptive petrography of the igneous rocks. Geologiska Föreningen i Stockholm Förhandlingar. v. 1.

Le Maitre R.W. 2002. Igneous rocks: a classification and glossary of terms: Recommendations of the IUGS Subcommission on the Systematics of Igneous rocks. $2^{\text {a }}$ ed. Cambridge, Cambridge University Press.

MacDonald G.A., Katsura T. 1964. Chemical composition of Hawaiian lavas. Journal of Petrology, 5(1):82-133. https:/doi.org/10.1093/ petrology/5.1.82

Machado F.B., Nardy A.J.R., Oliveira M.A.F. 2007. Geologia e aspectos petrológicos das rochas intrusivas e efusivas mesozóicas de parte da borda leste da Bacia do Paraná no estado de São Paulo. Revista Brasileira de Geociências, 37(1):64-80.

Mantovani M.S.M., Cordani U.G., Roisenberg A. 1985. Geoquímica isotópica em vulcânicas ácidas da Bacia do Paraná e implicações genéticas associadas. Revista Brasileira de Geociências, 15:61-65.

Marques L.S., De Min A., Rocha-Júnior E.R.V., Babinski M., Bellieni G., Figueiredo A.M.G. 2017. Elemental and Sr-Nd-Pb isotope geochemistry of the Florianópolis Dyke Swarm (Paraná Magmatic Province): crustal contamination and mantle source constraints. Journal of Volcanology and Geothermal Research. http://dx.doi. org/10.1016/j.jvolgeores.2017.07.005

Marques L.S., Dupré B., Piccirillo E.M. 1999. Mantle source compositions of the Paraná Magmatic Province (southern Brazil): evidence from trace element and $\mathrm{Sr}-\mathrm{Nd}-\mathrm{Pb}$ isotope geochemistry. Journal of Geodynamics, 28(4-5):439-458. https://doi.org/10.1016/ S0264-3707(99)00020-4

Marques L.S., Ernesto M. 2004. O magmatismo toleítico da Bacia do Paraná. In: Mantesso-Neto V., Bartorelli A., Carneiro C.D.R., BritoNeves B.B. (Eds.). Geologia do Continente Sul-Americano: evolução da obra de Fernando Flávio Marques de Almeida, p. 245-263. São Paulo, Beca.

Marques L.S., Rocha-Júnior E.R.V., Babinski M., Carvas K.Z., Petronilho L.A., De Min A. 2016. Lead isotope constraints on the mantle sources involved in the genesis of Mesozoic high-Ti tholeiite dykes (Urubici type) from the São Francisco Craton (Southern Espinhaço, Brazil). Brazilian Journal of Geology, 46:105-122. http://dx.doi. org/10.1590/2317-4889201620150010

Milani E.J. 2004. Comentários sobre a origem e evolução tectônica da Bacia do Paraná. In: Mantesso-Neto V., Bartorelli A., Carneiro C.D.R., Brito-Neves B.B. (Eds.), Geologia do Continente Sul-Americano: evolução da obra de Fernando Flávio Marques de Almeida, p. 265-291. São Paulo, Beca.

Milani E.J., Melo J.H.G, Souza P.A., Fernandes L.A., França A.B. 2007. Bacia do Paraná. Boletim de Geociências da Petrobras, 15(2):265-287.

Morimoto N. 1988. Nomenclature of pyroxenes. Mineralogy and Petrology, 39(1):55-76. https://doi.org/10.1007/BF01226262

O’Hara M.J. 1977. Geochemical evolution during fractional crystallisation of a periodically refilled magma chamber. Nature, 266:503-507. https://doi.org/10.1038/266503a0

Pacheco F.E.R.C., Caxito F.A., Moraes L.C., Marangoni Y.R., Santos P.R.S, Pedrosa-Soares A.C. 2018. Basaltic ring structures of the Serra Geral Formation at the southern Triângulo Mineiro, Água Vermelha region, Brazil. Journal of Volcanology and Geothermal Research, 355:136-148. http://dx.doi.org/10.1016/j.jvolgeores.2017.06.019

Pearce J.A. 2008. Geochemical fingerprinting of oceanic basalts with applications to ophiolite classification and the search for Archean oceanic crust. Lithos, 100(1):14-48. https://doi.org/10.1016/j. lithos.2007.06.016

Peate D.W. 1997. The Paraná-Etendeka Province. In: Mahoney J.J., Coffin M.F., eds., Large igneous provinces: continental, oceanic, and planetary flood volcanism, 217-245. Washington, D.C., American Geophysical Union.

Peate D.W., Hawkesworth C.J., Mantovani M.S. 1992. Chemical stratigraphy of the Paraná lavas (South America): classification of magma types and their spatial distribution. Bulletin of Volcanology, 55(1):119-139. https://doi.org/10.1007/BF00301125

Piccirillo E.M., Melfi A.J. 1988. The Mesozoic flood volcanism of the Paraná basin: petrogenetic and geophysical aspects. São Paulo, Instituto Astronômico e Geofísico da Universidade de São Paulo, 600 p.

Pik R., Deniel C., Coulon C., Yirgu G., Marty B. 1999. Isotopic and trace element signatures of Ethiopian flood basalts: evidence for plume-lithosphere interactions. Geochimica et Cosmochimica Acta, 63(15):2263-2279. https://doi.org/10.1016/ S0016-7037(99)00141-6

Pinto V.M., Hartmann L.A. 2011. Flow-by-flow chemical stratigraphy and evolution of thirteen Serra Geral Group basalt flows from Vista Alegre, southernmost Brazil. Anais da Academia Brasileira de Ciências, 83(2):425-440. http://dx.doi.org/10.1590/ S0001-37652011000200006

Reichow M.K., Saunders A.D., White R.V., Al'Mukhamedov A.I., Medvedev A.Y. 2005. Geochemistry and petrogenesis of basalts from the West Siberian Basin: an extension of the Permo-Triassic Siberian Traps, Russia. Lithos, 79(3-4):425-452. https://doi.org/10.1016/j. lithos.2004.09.011

Renne P.R., Glen J.M., Milner S.C., Duncan A.R. 1996. Age of Etendeka flood volcanism and associated intrusions in southwestern Africa. Geology, 24(7):659-662. https://doi. org/10.1130/0091-7613(1996)024\%3C0659:AOEFVA\%3E2.3.CO;2

Rocha-Júnior E.R., Marques L.S., Babinski M., Nardy A.J., Figueiredo A.M., Machado F.B. 2013. Sr-Nd-Pb isotopic constraints on the nature of the mantle sources involved in the genesis of the high-Ti tholeiites from northern Paraná Continental Flood Basalts (Brazil). Journal of South American Earth Sciences, 46:9-25.

Seer H.J., Moraes L.C., Carneiro M.A. 2011. Geologia e litogeoquímica dos diques toleíticos ATi vinculados aos lineamentos magnéticos de direção NW do Arco do Alto Paranaíba em Abadia dos Dourados, MG. In: Simpósio Vulcanismo e Ambientes Associados, 5. Resumos... Sociedade Brasileira de Geologia. CD-ROM.

Spera F.J., Bohrson W.A. 2004. Open-system magma chamber evolution: An energy-constrained geochemical model incorporating the effects of concurrent eruption, recharge, variable assimilation and fractional crystallization (EC-E' RA $\chi \mathrm{FC}$ ). Journal of Petrology, 45(12):2459-2480. https://doi.org/10.1093/petrology/egh072

Sun S.S., McDonough W.S. 1989. Chemical and isotopic systematics of oceanic basalts: implications for mantle composition and processes. Geological Society, London, Special Publications, 42(1):313-345. https://doi.org/10.1144/GSL.SP.1989.042.01.19

Swanson D.A., Wright T.L., Helz R.T. 1975. Linear vent systems and estimated rates of magma production and eruption for the Yakima Basalt on the Columbia Plateau. American Journal of Science, 275(8):877-905. https://doi.org/10.2475/ajs.275.8.877

Thompson R.N., Morrison M.A., Hendry G.L., Parry S.J., Simpson P.R., Hutchison R., O'Hara M.J. 1984. An Assessment of the Relative Roles of Crust and Mantle in Magma Genesis: An Elemental Approach [and Discussion]. Philosophical Transactions of the Royal Society of London A: Mathematical, Physical and Engineering Sciences, 310(1514):549-590. https://doi. org/10.1098/rsta.1984.0008

Turner S., Hawkesworth C. 1995. The nature of the sub-continental mantle: constraints from the major-element composition of continental flood basalts. Chemical Geology, 120(3-4), 295-314. 
Turner S., Hawkesworth C., Gallagher K., Stewart K., Peate D., Mantovani M. 1996. Mantle plumes, flood basalts, and thermal models for melt generation beneath continents: Assessment of a conductive heating model and application to the Paraná. Journal of Geophysical Research: Solid Earth, 101(B5), 11503-11518.

Wang C.Y., Zhou M.F., Qi L. 2007. Permian flood basalts and mafic intrusions in the Jinping (SW China)-Song Da (northern Vietnam) district: mantle sources, crustal contamination and sulfide segregation. Chemical Geology, 243(3):317-343. https://doi.org/10.1016/j.chemgeo.2007.05.017

Wang X.C., Li X.H., Li W.X., Li Z.X., Ying L., Yang Y.H., Liang X.R., Tu X.L. 2008. The Bikou basalts in the northwestern Yangtze block,
South China: Remnants of 820-810 Ma continental flood basalts? Geological Society of America Bulletin, 120(11-12):1478-1492. http://doi.org/10.1130/B26310.1

Webster T.L., Murphy J.B., Gosse J.C. 2006. Mapping subtle structures with light detection and ranging (LIDAR): flow units and phreatomagmatic rootless cones in the North Mountain Basalt, Nova Scotia. Canadian Journal of Earth Sciences, 43(2):157-176. https://doi.org/10.1139/e05-099

Zalán P.V., Wolff S., Conceição J.D.J., Astolf M.A., Vieira I.S., Zanotto O.A., Marques A. 1991. Tectonics and sedimentation of the Paraná Basin. In: International Gondwana Symposium (v. 7, p. 83-117). 